Louisiana State University

LSU Digital Commons

$4-2-2018$

\title{
Articulatory Kinematics During Stop Closure in Speakers with Parkinson's Disease
}

Austin Ross Thompson

Louisiana State University and Agricultural and Mechanical College

Follow this and additional works at: https://digitalcommons.lsu.edu/gradschool_theses

Part of the Speech and Hearing Science Commons, and the Speech Pathology and Audiology Commons

\section{Recommended Citation}

Thompson, Austin Ross, "Articulatory Kinematics During Stop Closure in Speakers with Parkinson's Disease" (2018). LSU Master's Theses. 4687.

https://digitalcommons.Isu.edu/gradschool_theses/4687

This Thesis is brought to you for free and open access by the Graduate School at LSU Digital Commons. It has been accepted for inclusion in LSU Master's Theses by an authorized graduate school editor of LSU Digital Commons. For more information, please contact gradetd@lsu.edu. 


\title{
ARTICULATORY KINEMATICS DURING STOP CLOSURE IN SPEAKERS WITH PARKINSON'S DISEASE
}

\author{
A Thesis \\ Submitted to the Graduate Faculty of the \\ Louisiana State University and \\ Agricultural and Mechanical College \\ in partial fulfillment of the \\ requirements for the degree of \\ Master of Arts \\ in
}

The Department of Communication Sciences and Disorders

by

Austin Ross Thompson

B.A., Louisiana State University, 2016

May 2018 


\section{Table of Contents}

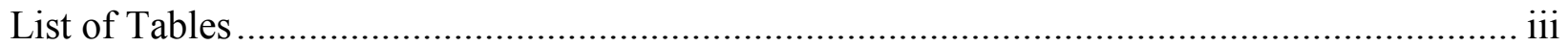

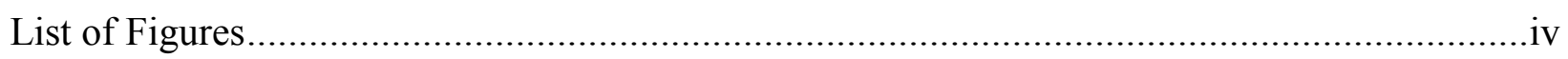

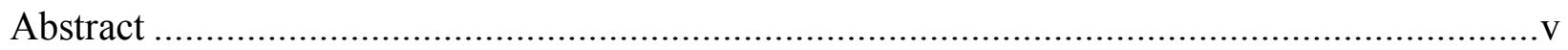

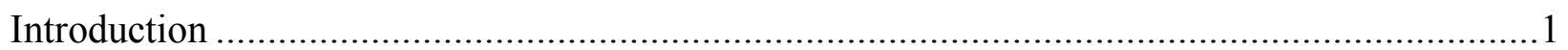

Parkinson's Disease: Definition, Prevalence, and Etiologies ......................................1

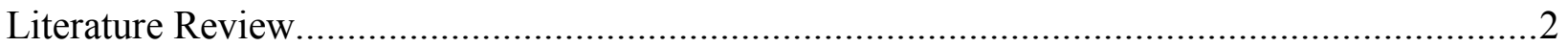

Speech Characteristics of Speakers with PD ........................................................2

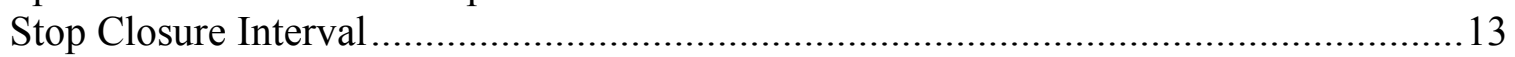

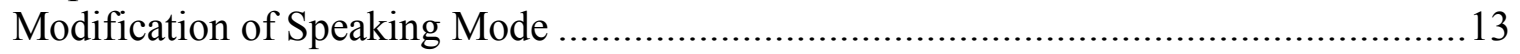

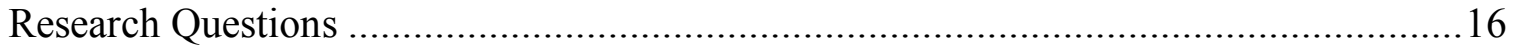

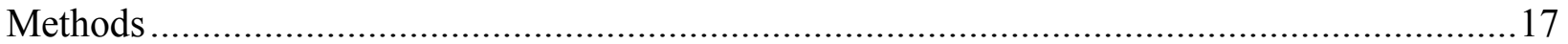

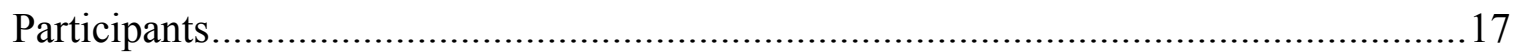

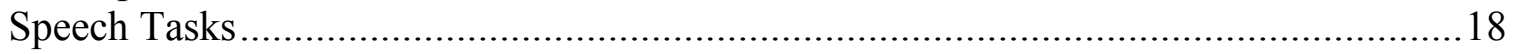

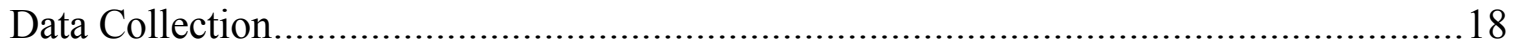

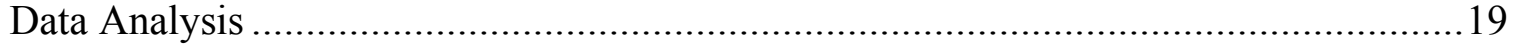

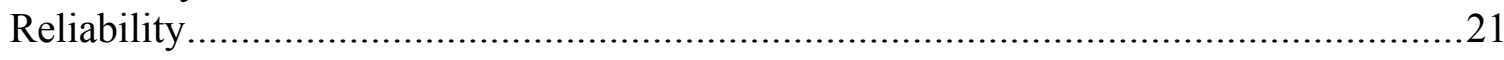

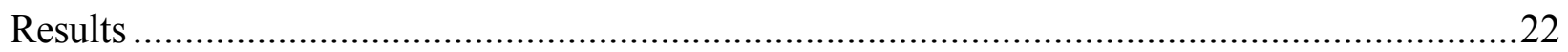

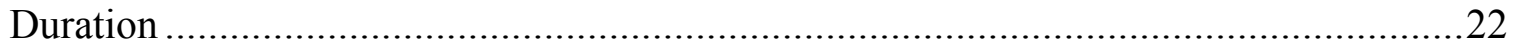

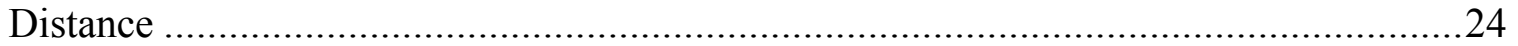

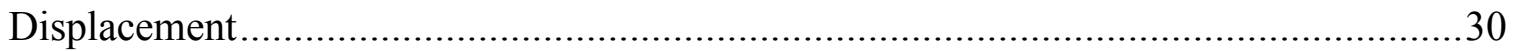

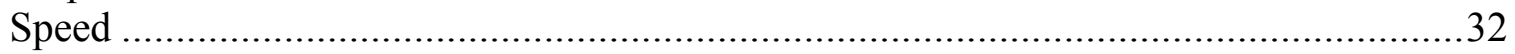

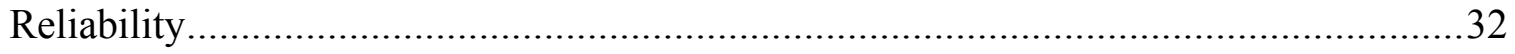

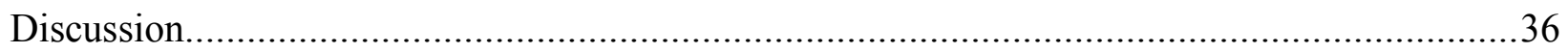

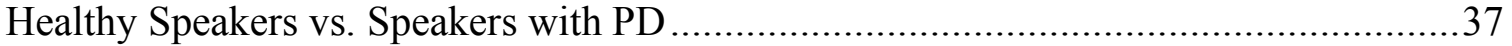

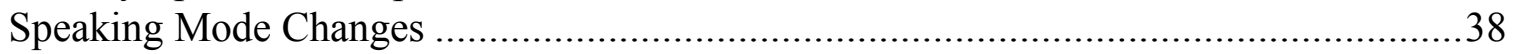

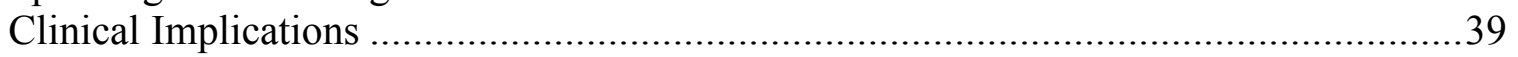

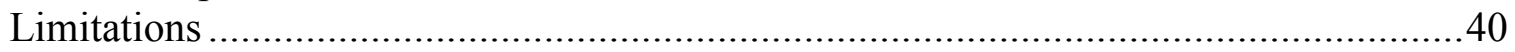

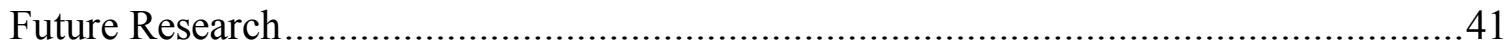

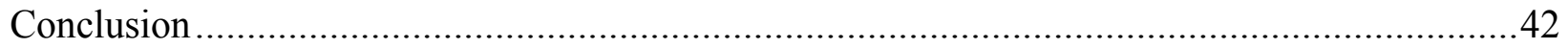

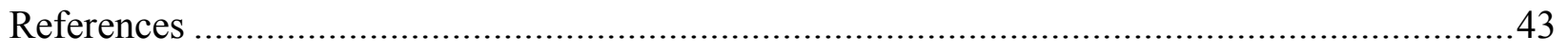

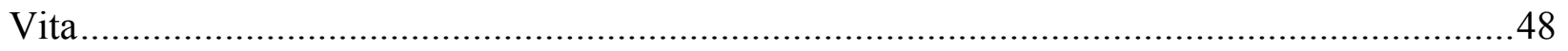




\section{List of Tables}

Table 1. Summary of Primary Speech Characteristics of Speakers with

Parkinson's Disease

Table 2. General Characteristics of Participants with Parkinson's Disease and

Healthy Control Participants.

Table 3. Articulatory Measurements for Each Articulator Produced by

Participants Using a Conversational Speaking Mode

Table 4. Articulatory Measurements for Each Articulator Produced by

Participants Using a More Clear Speaking Mode

Table 5. Articulatory Measurements for Each Articulator Produced by

Participants Using a Less Clear Speaking Mode 35 


\section{List of Figures}

Figure 1. A schematic representation of distance and displacement measures ........................9

Figure 2. Identification of the closure interval of bilabial stops......................................20

Figure 3. Comparisons of stop closure duration and voice onset time for the stop consonant $/ \mathrm{b} /$ produced by speakers with PD and healthy controls with a conversational speaking mode. .23

Figure 4. Kinematic and acoustic signals during the production of "Buy Bobby a puppy" spoken by a healthy speaker and a speaker with PD

Figure 5. Comparison of the UL, LL, TF, and TB articulators during a conversational production of "buy.".

Figure 6. Comparison of UL and LL closure onset and offset movement of consonant $/ \mathrm{b} /$ produced by speakers HC 2 and PD 2

Figure 7. Comparison of UL and LL displacement during the stop closure of consonant $/ \mathrm{b} /$ produced by speakers $\mathrm{HC} 2$ and PD 3 


\begin{abstract}
Purpose: The goal of this exploratory study was (a) to investigate the differences in articulatory movements during the closure phase of bilabial stop consonants with respect to distance, displacement, and timing of motion between individuals with Parkinson's Disease (PD) and healthy controls; and (b) to investigate changes in articulatory movements of speakers with PD when they voluntarily vary the degree of speech intelligibility.
\end{abstract}

Methods: Six participants, 4 PD and 2 healthy control (HC) speakers, participated in this study. The stimulus was a sentence containing several bilabial stop consonants (i.e., “Buy Bobby a puppy”). Movement data were collected using the Wave Speech Research System (NDI, Canada). Movement measures included duration, distance, and displacement and speed of the tongue front, tongue back, upper lip, lower lip, and jaw.

Results: Speakers with PD and HC speakers produced observable articulatory differences during the stop closure of bilabial stops. Generally, the PD group produced smaller articulatory movement and had longer closure durations than the HC group. Regarding changes in speaking mode, the two groups made observable, but different articulatory changes during the stop closure. For more clear speech, both groups made greater articulatory movements and decreased the stop closure duration. For less clear speech, the HC group demonstrated reduced articulatory movements and longer closure durations whereas the PD group made greater articulatory movements and longer closure durations.

Discussion: The findings of this study revealed several articulatory differences during the stop closure between the two speaking groups. For more clear speaking conditions, speakers in the PD group can successfully compensate for reduced articulatory movement by producing exaggerated lower lip and jaw movement. These findings support the use of more clear speaking 
modifications as a therapeutic technique to elicit better articulatory movement among speakers with PD. However, it also appears the PD group has difficulty producing fine motor articulatory changes (e.g., less clear speech). 


\section{Introduction}

\section{Parkinson's Disease: Definition, Prevalence, and Etiologies}

Parkinson's Disease (PD) is a progressive neurodegenerative disease that affects $1 \%$ to $2 \%$ of the population over the age of 50 (Jang, Boltz, Webster, \& Smeyne, 2008). PD is characterized by a dysfunction of the basal ganglia caused by a loss of nerve cells in the substantia nigra and decreased dopamine content in the striatum (Duffy, 2013). Motor impairments, such as increased rigidity of the extremities, bradykinesia, and a festinating gait are salient features of PD (Jang, Boltz, Webster, \& Smeyne, 2008). However, PD may also present cognitive deficits, swallowing difficulties, and speech impairments (Darley, Aronson, \& Brown, 1969a; Hochstadt, Nakano, Lieberman, \& Friedman, 2005; Duffy, 2013). With an average life expectancy of 15 years post-diagnosis, it is necessary that individuals with PD receive treatment for their various deficits (Jang, Boltz, Webster, \& Smeyne, 2008).

Dysarthria develops in approximately $90 \%$ of individuals with PD a few years post-onset (Müller et al., 2001). Of the various types of dysarthria, hypokinetic dysarthria is the most common among individuals with PD and is often referred to as "the dysarthria of PD" (Duffy, 2013). The following sections summarize the main findings from three types of speech measurement approaches: auditory-perceptual, acoustic, and kinematic. All three measurement techniques provide unique and meaningful information to our understanding of dysarthric speech. 


\section{Literature Review}

\section{Speech Characteristics of Speakers with PD}

Auditory-perceptual measurements. The Mayo Clinic's dysarthria studies, conducted by Darley, Aronson, and Brown (1969a, 1969b), serve as the foundation for identifying and classifying dysarthria. Before these studies, dysarthria was broadly defined as "imperfect articulation in speech" and failed to describe the unique speech and voice features associated with specific neurological disorders (Dorland, 1965). From this study, Darley et al. (1969a) observed a correlation between perceptual speech characteristics and the patient's neuroanatomical deficits. Furthermore, unique dysarthria patterns were observed among the six neurologic groups (spastic, flaccid, hypokinetic, hyperkinetic, ataxic, and mixed). Based on their neuropathology-oriented system, the speech of individuals with PD was described as hypokinetic. Perceptual, acoustic, and kinematic characteristics of speech produced by individuals with hypokinetic dysarthria secondary to PD are summarized in Table 1 (page 12).

Range of movement. The term hypokinetic means 'reduced movement'. Not surprisingly, most speech characteristics (perceptual, acoustic, and kinematic characteristics) are believed to stem from this neuropathology due to the rigidity associated with PD. Darley et al. (1969b) noted a reduced range of motion for individual movements made by speakers with PD. However, they observed a greater reduction of movement during repetitive movements (e.g., diadochokinetic tasks).

Precision of movement. Hypokinetic speech is further described as having imprecise articulation (Darley et al., 1969a). Logemann and Fisher (1981) examined the misarticulations of speakers with PD. They found the most frequent articulatory errors involved changes in the manner of stops, fricatives, and affricates. Stop consonants, as well as the stop portion of 
affricates, were often perceived as fricatives. Furthermore, fricatives were perceived as less "sharp" and less forceful. These manner errors were presumed to be caused by insufficient and imprecise articulatory movements; stops and affricates were perceived as fricative-like due to insufficient oral closure and fricatives were perceived as less "sharp" due to inadequate tongue elevation to achieve a constriction of the airway.

Rate of movement. Within the dysarthria classification system created by Darley et al. (1969a), hypokinetic dysarthria is the only type of dysarthria to demonstrate prosodic features such as short rushes of speech, relatively fast speaking rate, and festinating speech. The accelerated rate combined with reduced movement results in a "blurred" quality of speech (Duffy, 2013). Although Darley et al. (1969a) describes normal-to-fast rates of speech as a salient feature of hypokinetic speech, others have reported comparable perceptual speech rates between speakers with PD and healthy controls (Canter, 1963). In addition to the above perceptual speech characteristics, Darley et al. (1969a) described the prosodic characteristics of hypokinetic dysarthria as monopitch, reduced stress, monoloudness, inappropriate silences, harsh voice, breathy voice, low pitch, and variable rate.

Acoustic measurements. Acoustic measurements have been used in a way to support auditory-perceptual assessments of speech characteristics of various types of dysarthria including hypokinetic with improved reliability and accuracy compared to the aforementioned perceptual approach. Similar to perceptual measurements, acoustic measurements examine the speech signal; however, unlike auditory-perceptual measurements, acoustic measurements objectively quantify speech signals and examine various speech subsystems (i.e., respiration, phonation, resonance, articulation, and prosody; Duffy, 2013). 
Range of movement. Numerous studies have explored various acoustic measurements that are believed to be indicative of a reduced range of movement among individuals with PD (e.g., Goberman \& Elmer, 2005; Sapir, Ramig, Spielman, \& Fox, 2010; Skodda, Visser, \& Schlegal, 2011). Two acoustic measures, vowel space area, and formant centralization ratio have been used in the literature to infer the degree of reduced articulatory motion (hence, reduced acoustic contrasts) for vowel production.

Vowel space area (VSA). VSA, sometimes called acoustic vowel space, is an acoustic measurement believed to have a relationship with speech intelligibility and disease severity (Kent \& Kim, 2003; Bradlow et al., 1996). VSA is typically computed using the F1-F2 values of corner vowels to create (a) a quadrilateral VSA using the corner vowels /i/, /u/, /a/, and /æ/; or (b) a triangular VSA using the corner vowels /i/, /u/, and /a/ (Kent \& Kim, 2003). The size of the VSA is believed to be correlated with the articulatory productions of the corner vowels; a greater VSA suggests greater distinctiveness of articulatory positions for corner vowel production (Weismer, Yunusova, \& Bunton, 2012).

Many studies have reported a correlation between speech intelligibility and VSA. Bradlow, Torretta, and Pisoni (1996) examined the correlation between acoustically measured speaker characteristics (e.g., fundamental frequency [F0], VSA, and speaking rate) and speech intelligibility of healthy speakers. They found speakers with expanded VSAs were more intelligible than speakers with reduced VSAs. Many believe that a decreased VSA is caused by articulatory undershoot (Linblom, 1963), or reduced range of articulatory movement, which results in a decreased working space. Furthermore, researchers have found similar positive correlations between VSA and speech intelligibility in individuals with PD with reports of 
decreased VSAs among individuals with PD relative to healthy speakers (e.g., Goberman \& Elmer, 2005).

Despite many findings suggesting decreased VSA among individuals with PD, other reports have failed to find statistically significant differences between the VSA of individuals with hypokinetic dysarthria and healthy speakers (e.g., Sapir, Spielman, Ramig, Story, \& Fox, 2007; Weismer et al., 2001). Due to the conflicting reports, some researchers have proposed alternative acoustic measurements to quantify the reduction of movement among individuals with hypokinetic dysarthria.

Formant centralization ratio (FCR). Sapir, Ramig, Spielman, and Fox (2010) proposed FCR as a more reliable alternative to VSA for differentiating between healthy speakers and speakers with PD. FCR was designed to maximize sensitivity to vowel centralization and is expressed as $(F 2 u+F 2 a+F 1 i+F 1 u) /(F 2 i+F 1 a)$. The FCR is expressed as a ratio and is designed to reduce interspeaker variability in formant frequencies. Ratios greater than one are believed to be indicative of vowel centralization. The FCR was found to be sensitive and capable of differentiating hypokinetic speech from healthy speech (Sapir, Ramig, Speilman, \& Fox, 2010; Skodda et al., 2011).

In a recent study, Lansford and Liss (2014) examined the effectiveness of various acoustic measurements (including VSA and FCR) in distinguishing healthy speech from dysarthric speech. Of the nine acoustic measurements examined, they found FCR to have the least amount of healthy control misclassifications. However, FCR also had the most dysarthric misclassifications. This finding indicates that FCR has good specificity but poor sensitivity. In comparison, quadrilateral VSA classified speakers with 80\% accuracy. Lansford and Liss (2014) 
concluded that the use of several acoustic measurements are more effective with dysarthria identification than any single acoustic measurement used in isolation.

Precision of movement. Imprecise articulation is one of the deviant perceptual speech characteristics used by Darley et al. (1969a) to describe the articulation of hypokinetic speech. As previously mentioned, articulatory undershoot is believed to be the inability to reach or sustain articulatory targets (Duffy, 2013). Articulatory undershoot is commonly interpreted as a deficit with the precision of articulatory movements.

Spirantization. In typical stop consonant production, there is complete articulatory obstruction of airflow during the closure, or silent interval. However, among speakers with PD, acoustic analyses have revealed low intensity, fricative-like, aperiodic noise during the closure interval (Ramig, Titze, Scherer, \& Ringel, 1988; Weismer et al., 2012). This acoustic feature is known as spirantization. These findings validate the perceptual observations made by Logemann and Fisher (1981) and further suggest speakers with PD often fail to produce a sufficient oral closure for stop production. Spirantization is commonly interpreted as evidence of imprecise articulatory movements (Duffy, 2013).

Rate of movement. Several studies have examined acoustic parameters that are believed to be correlated with the rate of articulatory movement. The speech rate of speakers with hypokinetic dysarthria secondary to PD has been the subject of many perceptual and acoustic studies (e.g., Canter, 1963; McRae, Tjaden, \& Schoonings, 2002; Tjaden \& Wilding, 2004; and Nishio \& Niimi, 2001). In fact, hypokinetic dysarthria secondary to PD is the only type of dysarthria perceptually characterized to have a normal to fast rate of speech (Darley et al., 1969a; but see Canter, 1963). Interestingly, despite having the perception of accelerated speech, speakers with hypokinetic dysarthria have been found to articulate at an equal rate to healthy 
speakers (Nishio \& Niimi, 2001). Furthermore, it has been suggested that the perception of accelerated hypokinetic speech is not due to an increase in articulatory movement, but rather due to the reduced range of articulatory movement (Kent \& Rosenbek, 1982).

F2 slopes. Formant transitions, or the changes in formant frequencies as a function of time, reflect modifications in vocal tract configuration throughout an utterance. Several acoustic measurements can be obtained from formant transitions, including transition durations (i.e., the length of frequency change), transition extents (i.e., amount of frequency change), and transition rate or slope (i.e., the rate of frequency change; Weismer, Yunusova, \& Bunton, 2012).

Transition slopes, specifically F2 slopes of diphthongs, have been of particular interest among speakers with dysarthria. In healthy speakers, diphthongs require large changes in vocal tract configuration resulting in steep F2 slopes. However, F2 slopes have been found to be shallower among speakers with dysarthria, including speakers with PD, than in healthy speakers (Kim, Weismer, Kent, \& Duffy, 2009). Shallow F2 slopes are frequently interpreted as evidence of reduced rate or speed of change in vocal tract configuration. Furthermore, F2 slopes have been found to correlate with measures of speech intelligibility (Kim, Kent, \& Weismer, 2011).

Kinematic measurements. Researchers have explored various measurements to establish reliable methods for identifying and describing dysarthria. The direct measurement of the articulatory movement of individual or collective articulators (i.e., tongue, lips, jaw) is an advantage of using articulatory kinematic methods over traditional perceptual and acoustic methods (Yunusova, Weismer, Westbury, \& Lindstrom, 2008). Electromagnetic articulography (EMA), and other kinematic measurement techniques directly investigate articulatory movements, rather than speech signals. Speech kinematic research has been primarily utilized to investigate and describe the range, speed, and variability of articulatory movement. Speech 
movement research has been used in healthy speakers and speakers with various communication disorders including dysarthria, apraxia of speech, and fluency disorders (e.g., Forrest, Weismer, \& Turner, 1989; Smith \& Kleinow, 2000; Walsh \& Smith, 2012; Yunusova, Weismer, Westbury, \& Lindstrom, 2008).

Dysarthria has been the subject of much speech kinematic research. This area of research attempts to describe the movements of specific articulators to inform and guide the selection of effective clinical treatment for patients with dysarthria. However, most studies investigating movements of dysarthric speech have focused on speakers with mixed spastic-flaccid dysarthria secondary to ALS despite having a lower incidence than PD with rates of 1.2-1.8/100,000 and 3.59-132.72/100,00, respectively (Hirsch, Jette, Frolkis, Steeves, \& Pringsheim, 2016; Wang, Kothalkar, Cao, \& Heitzman, 2016). More research needs to focus on the articulatory movements of speakers with PD.

Range of movement. In the kinematic literature, the range of articulatory movement has been measured using two parameters: (1) distance (i.e., the length of movement) and (2) displacement (i.e., the Euclidean distance between the onset and offset of the movement; see Figure 1). Several studies have reported decreased articulatory movement among speakers with PD compared to their healthy, age-matched peers (e.g., Darling \& Huber, 2011; Forrest \& Weismer, 1995; Walsh \& Smith, 2012). Although, others have found comparable ranges of movement between speakers with PD and healthy, age-matched peers (Yunusova, Weismer, Westbury, \& Lindstrom, 2008). 


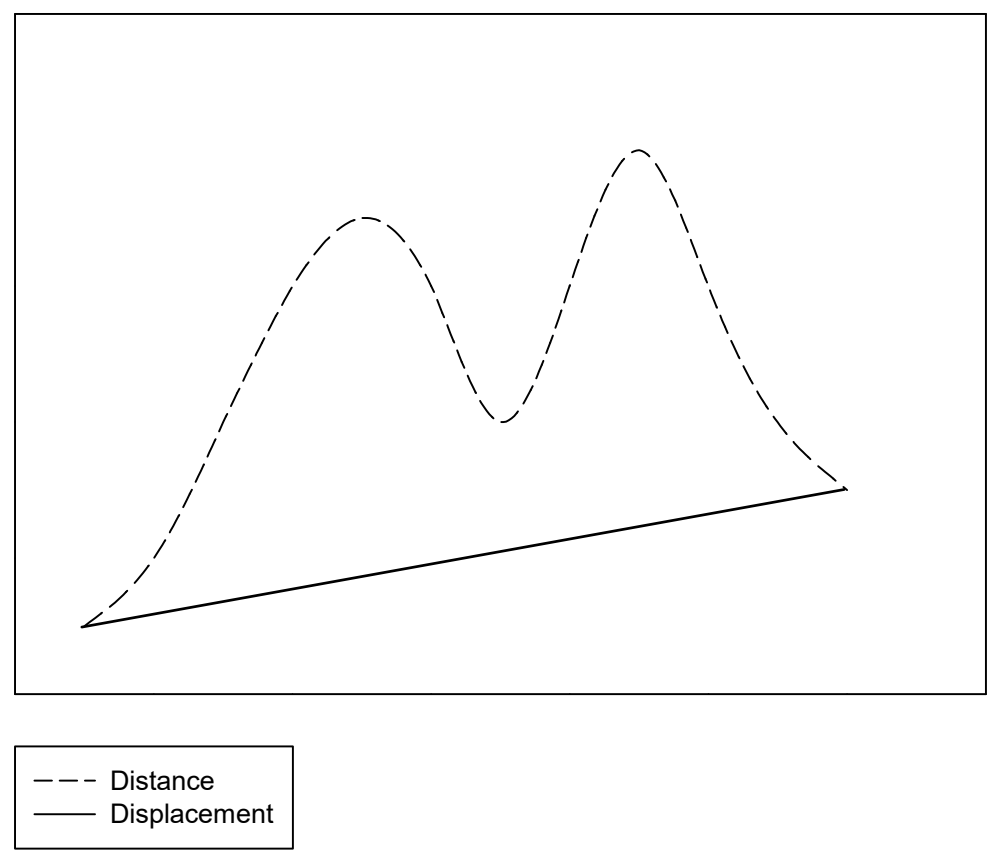

Figure 1. A schematic representation of distance and displacement measures.

Precision of movement. Measures of stability have served as indicators of articulatory precision. The lip aperture (LA) variability index is a measure of trial-to-trial variability of the upper lip, lower lip, and jaw movements. Compared to healthy, age-matched controls, speakers with PD have demonstrated significantly higher LA variability, and therefore less precise articulation (Walsh, 2007; Walsh \& Smith, 2011). However, other measures of stability have resulted in conflicting findings. Spatiotemporal index (STI), another measure of articulatory precision, examines the pattern and stability of movements (Smith, Johnson, McGillem, \& Goffman, 2000). A high STI is indicative of increased variability and less stable movement. Kleinow et al. (2001) found similar STI measures among speakers with PD and healthy agematched controls. This finding suggests speakers with PD and healthy, age-matched speakers have comparable variability and articulatory precision.

Rate of movement. The velocity of articulatory motion is the kinematic measurement used to describe the rate of motion. Consistent with acoustic findings of normal to slow 
articulatory rates among speakers with $\mathrm{PD}$, the kinematic literature reports reduced velocities of articulatory movement (Darling \& Huber, 2011; Forrest, Weismer, \& Turner, 1989; Walsh \& Smith, 2012). Although, these findings of reduced velocities have only been reported for labial and jaw movements. The velocity of lingual movement of speakers with PD appears to be absent from the literature.

Articulatory-to-acoustic and articulatory-to-perceptual relations. Much of our current knowledge about the speech movement of speakers with PD has been deduced from perceptual and acoustic findings. Perceptually, Darley et al. (1969) characterized the speech of individuals with PD as hypokinetic, or reduced in movement. Acoustic research has supported this characterization with many reports of reduced VSA among speakers with PD (e.g., Goberman \& Elmer, 2005; McRae, Tjaden, \& Schoonings, 2002; Sapir et al., 2007). These perceptual and acoustic findings have led to widely accepted articulatory implications despite a lack of empirical kinematic research to support these implications.

As previously mentioned, some kinematic research has observed reduced articulatory movement among speakers with PD (Darling \& Huber, 2011; Walsh \& Smith, 2012), whereas, others have failed to observe the same findings (Yunusova, Weismer, Westbury, \& Lindstrom, 2008). Furthermore, among healthy speakers, some studies have reported discrepancies between acoustic and kinematic measurements (e.g., Dromey, Jang, \& Hollis, 2013; Mefferd \& Green, 2010). These inconsistent findings, as well as reports of nonlinear relationships between acoustic and kinematic measurements, warrant the investigation of speech using kinematic measurement techniques; without studies examining the articulatory-to-acoustic relation of various measures, it is insufficient to assume the articulatory movement based on perceptual or acoustic measurements. 
Mefferd (2015) investigated the articulatory-to-acoustic relations of vowel contrasts in speakers with dysarthria secondary to ALS and PD. She examined the relationship between articulatory vowel contrasts (i.e., tongue displacement) and acoustic vowel contrasts (i.e., acoustic vowel distances). This study found a strong linear articulatory-to-acoustic relation among speakers with PD and a significantly weaker articulatory-to-acoustic relation among speakers with ALS. The findings of the study suggest that acoustic vowel contrasts can be used to accurately indicate the degree of tongue placements in speakers with PD, but not in speakers with ALS. 
Table 1. Summary of Primary Speech Characteristics of Speakers with Parkinson's Disease

\begin{tabular}{|c|c|c|c|}
\hline Speech Characteristics & Perceptual & Acoustic & Kinematic \\
\hline Range of movement & $\begin{array}{l}\text { Reduced range of } \\
\text { movement (Darley, } \\
\text { Aronson, \& Brown, } \\
\text { 1969) }\end{array}$ & $\begin{array}{l}\text { Reduced VSA } \\
\text { (suggesting reduced } \\
\text { movement; Goberman } \\
\text { \& Elmer, 2005; McRae, } \\
\text { Tjaden, \& Schoonings, } \\
\text { 2002; Sapir et al., } \\
\text { 2007) } \\
\text { Normal VSA } \\
\text { (suggesting normal } \\
\text { range of movement; } \\
\text { Sapir, Spielman, } \\
\text { Ramig, Story, \& Fox, } \\
\text { 2007; Weismer et al., } \\
\text { 2001) }\end{array}$ & $\begin{array}{l}\text { Reduced articulatory } \\
\text { movement (Darling \& } \\
\text { Huber, 2011; Walsh \& } \\
\text { Smith, 2012) } \\
\text { Normal movement } \\
\text { (Yunusova, Westbury, } \\
\text { \& Lindstrom, 2008) }\end{array}$ \\
\hline Precision of movement & $\begin{array}{l}\text { Imprecise consonants } \\
\text { (Darley, Aronson, \& } \\
\text { Brown, 1969) } \\
\text { Imprecise stops, } \\
\text { fricatives, and affricates } \\
\text { (Logemann \& Fisher, } \\
\text { 1981) }\end{array}$ & $\begin{array}{l}\text { Spirantization } \\
\text { (suggesting imprecise } \\
\text { articulation; Ramig, } \\
\text { Titze, Scherer, \& } \\
\text { Ringel, 1988; Weismer, } \\
\text { Yunusova, \& Bunton, } \\
\text { 2012) }\end{array}$ & $\begin{array}{l}\text { High LA variability } \\
\text { (suggesting imprecise } \\
\text { articulation; Walsh, } \\
\text { 2007) } \\
\text { Comparable STI } \\
\text { between PD and HC } \\
\text { (suggesting normal } \\
\text { articulatory precision; } \\
\text { Kleinow et al., 2001) }\end{array}$ \\
\hline Rate of movement & $\begin{array}{l}\text { Festinating speech, } \\
\text { variable rate (Darley, } \\
\text { Aronson, \& Brown, } \\
\text { 1969) } \\
\text { Normal rate (Canter, } \\
\text { 1963) }\end{array}$ & $\begin{array}{l}\text { Normal rate (Nishio \& } \\
\text { Niimi, 2001) } \\
\text { Shallow F2 slopes } \\
\text { (suggesting slow rate; } \\
\text { Kim, Weismer, Kent, \& } \\
\text { Duffy, 2009). }\end{array}$ & $\begin{array}{l}\text { Reduced velocity } \\
\text { (Forrest, Weismer, \& } \\
\text { Turner, 1989) }\end{array}$ \\
\hline
\end{tabular}

Note. $\mathrm{F} 2$ = second formant; $\mathrm{HC}=$ healthy control; LA variability = lip aperture variability; $\mathrm{PD}=$ Parkinson's disease; STI = spatiotemporal index; VSA = vowel space area 


\section{Stop Closure Interval}

Stop consonants (i.e., /p/, /b/, /t/,/d/, /k/, and /g/) are produced by creating a complete oral closure to stop airflow to allow for the build-up of intraoral pressure. After this brief closure, known as the stop closure interval, the pressure is released creating a burst of energy. As previously mentioned, acoustic studies have observed spirantization, or loose fricative-like energy, during the closure interval in people with PD (Weismer et al., 2012). These findings of abnormal closure intervals among speakers with PD warrant further investigations during this brief time interval. In the present study, a kinematic approach will be employed due to its advantage over perceptual and acoustic approaches in that articulatory events can be examined even when little-to-no acoustic signals are available.

Additionally, stop closure interval was selected in the study because of the high occurrence frequency of stop consonants. Stop consonants comprise $29 \%$ of all consonants and are highly likely to affect overall speech impression such as the ratings of speech intelligibility (Kent \& Kim, 2003). Despite their high occurrence within speech, there is little research focusing on the articulatory movements of stop closure intervals. Studies by Löfqvist and Gracco (1997, 2002) found meaningful coarticulatory gestures during the closure interval of a small number of healthy speakers. However, there remains a gap in the literature that directly examines the articulatory movements during the closure interval of English speaking individuals with PD. The purpose of the present study is to directly examine the articulatory movements during the closure interval of stop consonants among individuals with PD.

\section{Modification of Speaking Mode}

Many studies have examined the acoustic differences among speaking conditions of individuals with PD (e.g., Tjaden, 2004; Tjaden, Lam, \& Wilding, 2013). Speaking conditions 
have been investigated with the hopes of creating a theoretical foundation for the development of behavioral treatment methods for speakers with PD and other various types of dysarthria. Studies have explored the perceptual and acoustic changes of speech in habitual, slow, loud, and clear speaking conditions, however, few studies have examined the articulatory changes across speaking modes.

Slow speech. Increased rate of speech among speakers with PD has been well observed in the literature (e.g., Darley et al., 1969a; Nishio \& Niimi, 2001). As previously mentioned, despite the perception of increased speech rate, speakers with PD have been found to have articulatory rates similar to healthy speakers. It is hypothesized that the perceived increased speech rate is due to reduced articulatory motion (Kent \& Rosenbek, 1982). Therefore, it is believed slow speech may increase speech intelligibility by allowing the speakers more time to reach the target vocal tract configuration and minimize articulatory undershoot (Tjaden, Sussman, \& Wilding, 2014). Furthermore, reduced articulatory rate and expanded VSAs have been observed for slow speaking conditions among speakers with PD (Tjaden \& Wilding, 2004; Tjaden, Sussman, \& Wilding, 2014).

Loud speech. Perceptual and acoustic research has found that speakers with PD demonstrate reduced vocal loudness and monoloudness in habitual speaking contexts (e.g., Darley et al., 1969a; Ramig, Fox, \& Sapir, 2004). These findings have served as the foundation for behavioral treatment programs, such as Lee Silverman Voice Treatment (LSVT), a 1-month treatment program focusing on increasing loudness in speakers with PD (Sapir et al., 2007). Furthermore, loud speech has been found to elicit increased mean sound pressure levels effectively. However, it did not reduce the mean articulatory rate relative to habitual speech (Tjaden, Sussman, \& Wilding, 2014). 
Clear speech. Clear speech, a speaking condition characterized by exaggerated articulation, may serve as the optimal speaking condition for increasing speech intelligibility. Tjaden, Sussman, and Wilding (2014) examined the perceptual and acoustic speech changes among four speaking conditions: habitual, loud, slow, and clear. Like the slow condition, although to a lesser degree, the clear speaking condition resulted in reduced articulatory rate. Additionally, like the loud condition, to a lesser but comparable degree, the clear condition elicited increased sound pressure levels.

Furthermore, clear speech has been found to have F2 slopes (i.e., another acoustic measure of speech intelligibility) that were significantly different from habitual speaking conditions. However, the same differences were not observed between habitual speech and loud speech (Tjaden, Richards, Kuo, Wilding, \& Sussman, 2014). For these reasons, we chose to investigate clear speaking conditions in the current study.

In the current study, less clear speaking conditions were also investigated to assess the speakers' ability to modify their degree of speech intelligibility. To our knowledge, there are no studies assessing the ability of speakers with PD to modify their speech intelligibility with both more and less clear speaking conditions. The results of the current study may have clinical implications regarding the behavioral treatment possibilities for this population; if speakers can successfully alter their degree of speech intelligibility, clear speech may be a viable treatment technique. This study aims to examine the participant's articulatory movements as they voluntarily modify their degree of speech intelligibility (i.e., habitual, clear, and less clear speech). 


\section{Research Questions}

1. How do articulatory movements during the closure phase of bilabial stop consonants differ with respect to distance, displacement, duration, and speed of motion between individuals with PD and healthy controls?

We hypothesize that speakers with PD will demonstrate, in general, reduced articulatory movements. However, we expect that the extent of the reduction will vary across articulatory measurement points (tongue vs. lips) and movement parameters (distance, displacement, duration, speed).

2. Do speakers with PD exhibit changes in articulatory movements when they voluntarily vary the degree of speech intelligibility? If so, are the articulatory changes similar to the changes made by healthy controls?

We hypothesize that speakers with PD will be able to voluntarily modify their articulatory movement patterns in a similar pattern to healthy speakers, but to a lesser degree. 


\section{Methods}

\section{Participants}

Participants were four individuals with PD and two healthy control (HC) participants. PD participants were recruited from various PD social groups within the community. The participants ranged in age from 23-77 years $(M=60$ years). The mean age of the PD participants was 71 years. The mean age of the control group was 39. The time-since-disease onset among the PD group ranged from $1.5-15$ years $(M=6.88$ years $)$.

All participants were White, native speakers of English, and spoke a Southern American English (SAE) dialect. Participants reported no history of hearing loss, language difficulties, neurological disorders, or known structural abnormalities of the oral, pharyngeal, and respiratory mechanism. Medication was checked; however, it did not serve as inclusion criteria for the current study due to previous findings reporting medication having no significant effects on speech performance (Skodda, Visser, \& Schlegel, 2009). Finally, the participants reported no previous history of therapeutic treatment for their speech deficits.

Table 2. General Characteristics of Participants with Parkinson's Disease and Healthy Control Participants

\begin{tabular}{cccccc}
\hline Participant & Dx & Sex & Age & $\begin{array}{c}\text { Time since } \\
\text { onset (years) }\end{array}$ & Speech severity \\
\hline HC 1 & Healthy & M & 55 & - & - \\
HC 2 & Healthy & M & 23 & - & Severe \\
PD 1 & PD & M & 63 & 15 & Mild \\
PD 2 & PD & F & 73 & 1.5 & Moderate \\
PD 3 & PD & M & 71 & 2 & Moderate/Severe \\
PD 4 & PD & M & 77 & 9 &
\end{tabular}




\section{Speech Tasks}

The data investigated were collected as part of a larger study of articulatory movements produced by speakers with various motor speech disorders. The current study uses data collected from one of five speech tasks administered during a 2-hour data collection session. The speech task of interest was “Buy Bobby a puppy." This utterance was selected due to its high frequency of bilabial stop consonants. Speakers repeated the speech stimuli five times under three speaking conditions: conversational, more clear, and less clear. Speaking mode changes were elicited using a direct magnitude estimation approach. For the conversational condition, speakers were instructed to read the sentence in their normal reading voice. For the more clear speaking condition, speakers were instructed to read the sentence with twice the clarity of their normal speaking condition. For the less clear speaking condition, speakers were instructed to read the sentence with half the clarity of their normal speaking condition; speakers were asked to read "as if you were telling your friend a comment you did not want other people in the room to hear."

\section{Data Collection}

Kinematic data were collected using the Wave system (NDI, Canada), a 3D electromagnetic tracking system with the ability to track several $2 \mathrm{~mm}$-diameter five-degrees of freedom (5DOF) sensors. Using PariAcryl Oral Tissue Adhesive, a non-toxic dental surgical glue, six sensors were affixed to various articulators on the midsagittal plane. Articulators included tongue front (TF), tongue back (TB), and upper and lower lip (UL and LL). Two additional sensors served as references; one sensor was adhered to the labial surface of the lower central incisors to control for jaw movements relative to lingual and labial movements; the second sensor was attached to the bridge of plastic glasses without lenses to control for head movements relative to articulatory movements. Before kinematic data collection, a bite plate was 
obtained from each participant to define the maxillary occlusal and midsagittal planes. This information was used to develop a single quaternion rotation to transform the collected kinematic data into the anatomically-referenced space. Additionally, a palatal probe was used to trace the roof of the mouth to define the size and shape of the palate.

Acoustic and kinematic data were collected simultaneously in a sound-attenuating booth, with a sampling rate of $20 \mathrm{kHz}$ and 16-bit resolution. The acoustic data were segmented using TF32 (Milenkovic, 2004) to extract target kinematic data (i.e., kinematic data from the closure interval of stop consonants). An AKG C1000S microphone positioned approximately $30 \mathrm{~cm}$ from the speaker was used to record the speech stimuli.

\section{Data Analysis}

Closure interval identification. The onset and offset of the closure interval presented in each speech stimuli were calculated using acoustic and kinematic segmentation approaches. The offset of the closure interval was identified from the stop burst observed on the acoustic spectrogram and waveform. The onset of the closure interval was identified from the peak vertical velocity of the LL movement (see Figure 2). Traditionally, to determine the onset of the closure interval of stop consonants in the utterance initial position, measures of intraoral pressure are used to identify the sudden increase in pressure (see Löfqvist \& Gracco, 1997). A sudden increase in pressure is understood to be the moment of complete closure. However, Löfqvist and Gracco (1997) observed that at the moment of closure onset (as defined by the rise in intraoral pressure) the LL is at its peak vertical velocity. For this reason, we believe the peak vertical velocity of the LL is a valid indicator of closure onset. 


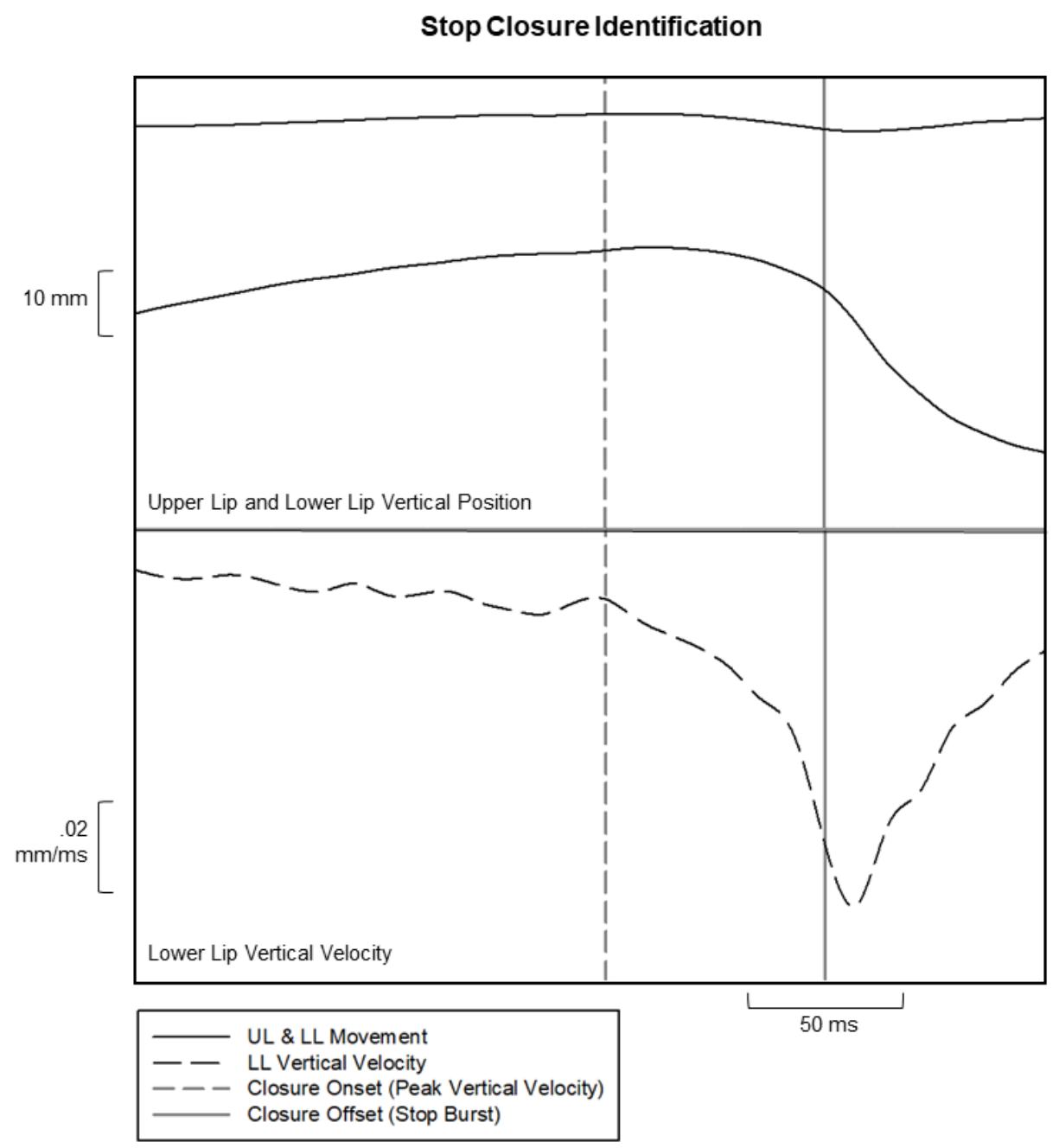

Figure 2. Identification of the closure interval for bilabial stops. UL = upper lip. LL = lower lip. The two vertical lines represent the onset and offset of the closure phase. The onset is identified from the peak vertical velocity of the LL movement. The offset is identified as the stop burst observed from the acoustic signal.

Kinematic data analysis. Kinematic data were analyzed with respect to distance, displacement, duration, and speed of the four articulatory measurement points (TF, TB, UL, and LL) during the closure interval of the utterance-initial bilabial stop consonant presented in the speech stimuli (i.e., “Buy Bobby a puppy”). 


\section{Reliability}

To establish intrameasurer reliability, the closure interval onset and offset from two participants, one speaker with PD and one control speaker was randomly selected to be measured again. The second measure occurred approximately three months after the first, and Pearson product moment correlation coefficients were obtained to measure the reliability. 


\section{Results}

The results of the study addressed the two research questions: (1) how do articulatory movements during the closure phase of stops consonants differ with respect to distance, displacement, duration, and speed of motion between the PD and HC groups; and (2) do speakers with PD exhibit changes in articulatory movements when they voluntarily vary the degree of speech intelligibility? If so, are the articulatory changes similar to the changes made by the $\mathrm{HC}$ group? Average measurements for duration, distance, displacement, and speed for each speaker are reported in Table 3, Table 4, and Table 5 (pages 33 - 35).

\section{Duration}

The duration of the stop consonant closure was defined as the time interval between the closure onset (i.e., peak velocity of the LL movement) to the closure offset (i.e., identified from the stop burst of the acoustic signal).

Healthy speakers vs. speakers with PD. With a conversational speaking mode, speakers with PD tended to demonstrate longer closure durations than the HC group, with average durations of $98.6 \mathrm{~ms}$ and $62.0 \mathrm{~ms}$, respectively. Participant PD 1, a speaker characterized by severe speech deficits, demonstrated considerably longer average closure durations (i.e., $152 \mathrm{~ms}$ ) compared to the other speakers with PD.

Our findings were inconsistent with previous reports of shorter closure durations in speakers with PD compared to their healthy peers (Cushine-Sparrow, Adams, Knowles, Leszcz, \& Jog, 2016). We believe this discrepancy warranted further investigation of the stop consonant, specifically the voice onset time (VOT). Previous studies have reported longer VOT durations for speakers with PD compared to HC group. Forrest, Weismer, and Turner (1989) reported an increased VOT for the consonant /b/ in the word-initial position in speakers with PD compared 
to HC group. Forrest et al. (1989) attributed the increased VOT in speakers with PD to their difficulty initiating and coordinating laryngeal movements. However, other studies have found shorter VOT among PD speakers compared to controls (see Flint, Black, Cambell-Taylor, Gailey, \& Levinton, 1992; Weismer, 1984). In the current study, further investigation of the VOT revealed findings similar to those reported by Forrest et al. (1989). Speakers with PD demonstrated longer and in some cases comparable, VOT for the consonant $/ \mathrm{b} /$ compared to the HC group (Figure 3). However, these findings of VOT should be interpreted with caution. As suggested by Fischer and Goberman (2010), VOT has been shown to vary as a function of rate and therefore must be examined independently of rate (i.e., a VOT ratio).
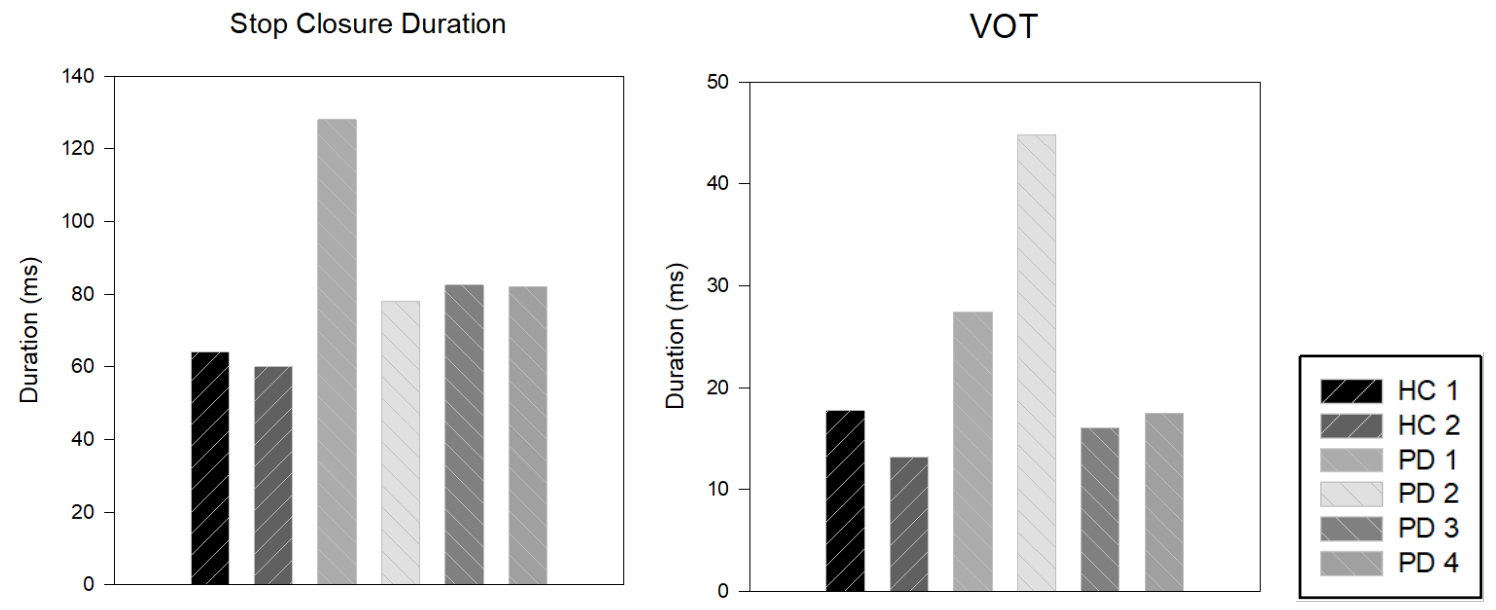

Figure 3. Comparisons of stop closure duration and voice onset time (VOT) for the stop consonant /b/ produced by speakers with $\mathrm{PD}$ and healthy controls with a conversational speaking mode. $\mathrm{HC}=$ healthy control; PD = Parkinson's disease.

Speaking mode changes. Relative to a conversational speaking mode, every speaker (except $\mathrm{HC} 2$ ) tended to decrease the stop closure duration during more clear speaking conditions, and increase the stop closure duration during less clear speaking conditions. The same trend was observed with VOT. For more clear speaking conditions, every speaker (except 
PD 2) shortened their VOT. Similarly, for less clear speaking conditions, every speaker (except HC 2) lengthened their VOT.

These unexpected findings warranted further investigation of the vowel duration following the stop consonant (i.e., /ai/). Consistent with previous studies (e.g., Tjaden, Lam, \& Wilding, 2013; Mefferd, 2017), we found that every speaker lengthened the vowel duration to produce a clear speaking condition and tended to shorten the vowel duration for less clear speaking conditions. These findings suggest that when a speaker attempts to produce more clear speech, they shorten the duration of the stop consonant (including the closure phase) to allow more time to produce the following vowel.

\section{Distance}

Distance was calculated as the cumulative articulatory movement for each sensor within the stop closure duration. Distance was normalized with respect to the stop closure duration. Data normalization was completed to ensure that speakers with longer closure durations were not misinterpreted as having greater distance measures.

Figure 4 displays the kinematic and acoustic signals of two speakers (HC 2 and PD 4) during the production of "Buy Bobby a puppy" using a conversational speaking mode. From the top panel to the bottom, Figure 4 presents the acoustic signal, the UL vertical position and velocity, the LL vertical position and velocity, and the lip aperture. The two vertical lines indicate the onset and offset of the stop closure of $/ \mathrm{b} /$; the onset was identified from the peak vertical velocity of the LL movement, and the offset was identified from the stop burst of the acoustic signal.

Healthy speakers vs. speakers with PD. Concerning labial movement, the average articulatory distances (combined vertical and horizontal movement) of the UL did not differ 
between the two speaking groups. However, further examination of vertical distance revealed the HC group tended to produce greater vertical UL movement compared to the PD group with average vertical distances of $2.07 \mathrm{~mm}$ and $1.19 \mathrm{~mm}$, respectively.
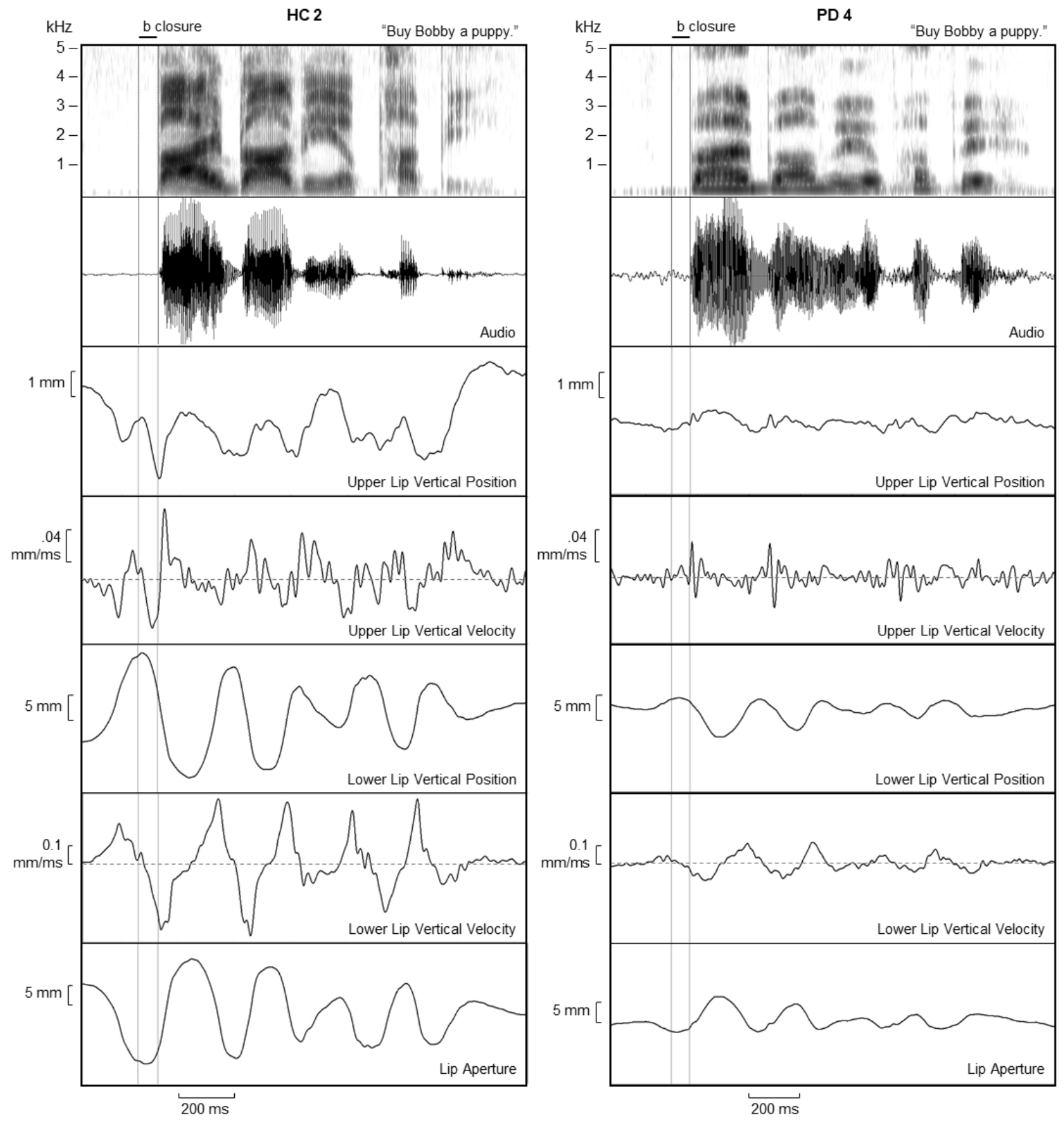

Figure 4. Kinematic and acoustic signals during the production of "Buy Bobby a puppy" spoken by a healthy speaker (HC 2; left) and a speaker with PD (PD 4; right). The two vertical lines represent the onset of the closure interval identified by the peak velocity and offset of the closure interval identified by the acoustic signals. 
Examination of the LL average articulatory distances (combined vertical and horizontal movement) revealed that the HC speakers demonstrated greater LL movement $(M=9.22 \mathrm{~mm})$ than the PD group $(M=6.04 \mathrm{~mm})$, as seen in Figure 4. Interestingly, the vertical LL movement was similar between the two speaking groups. However, the horizontal LL movement was greater in the HC group. These findings support the idea that speakers with PD may compensate for reduced movement by producing exaggerated vertical LL and jaw movement. However, it appears more difficult for speakers with PD to compensate for UL movement and horizontal LL movement.

Figure 5 demonstrates the articulatory differences of the UL, LL, TF, and TB articulators during a conversational production of "buy." The solid lines indicate the articulatory movement made during the stop closure. The dotted lines indicate the movement during the following diphthong, /aI/. Examination of the average lingual movement distances revealed the $\mathrm{HC}$ group (except PD 1) produced greater TF movement distances $(M=11.24 \mathrm{~mm})$ than the PD group $(M$ $=6.26 \mathrm{~mm})$ and, to a lesser degree, greater TB movement distances $(M=7.79 \mathrm{~mm})$ compared to the PD group $(M=6.32 \mathrm{~mm}$; see Figure 5$)$. Speaker PD 1 has had the greatest time since disease onset and was also perceived as having the most severe speech severity. We believe this speaker may be producing exaggerated lingual movement to compensate for his speech deficits.

Because the lingual movement was not decoupled from the jaw movement, we see the lingual movement in relation to the jaw movement. The lingual movement does not contribute to the stop production for consonant $/ \mathrm{b} /$, however, during the closure interval, the TF and TB sensors appear to begin the lingual motion needed to produce the following sound (i.e., diphthong /aI/ in "buy"). 
$\mathrm{HC} 2$

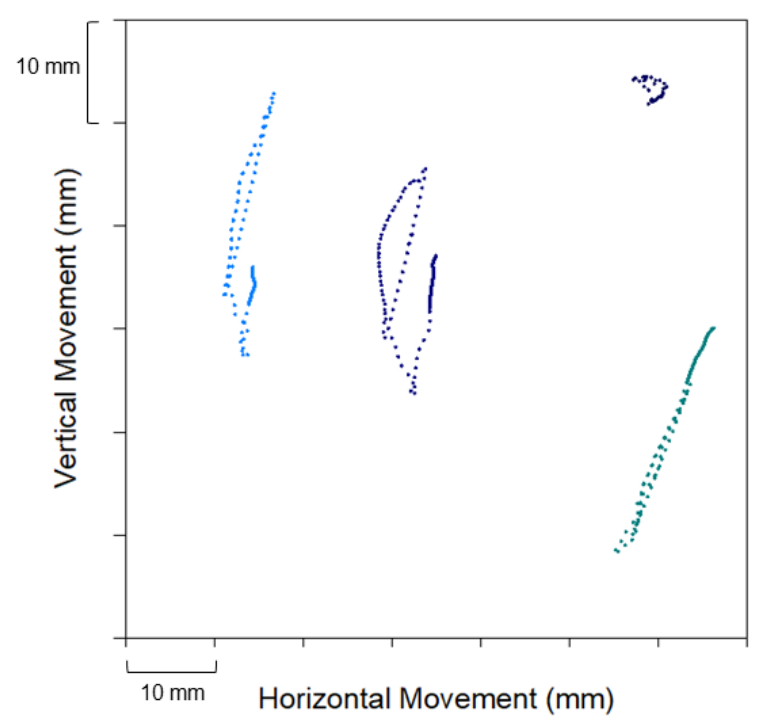

PD 3

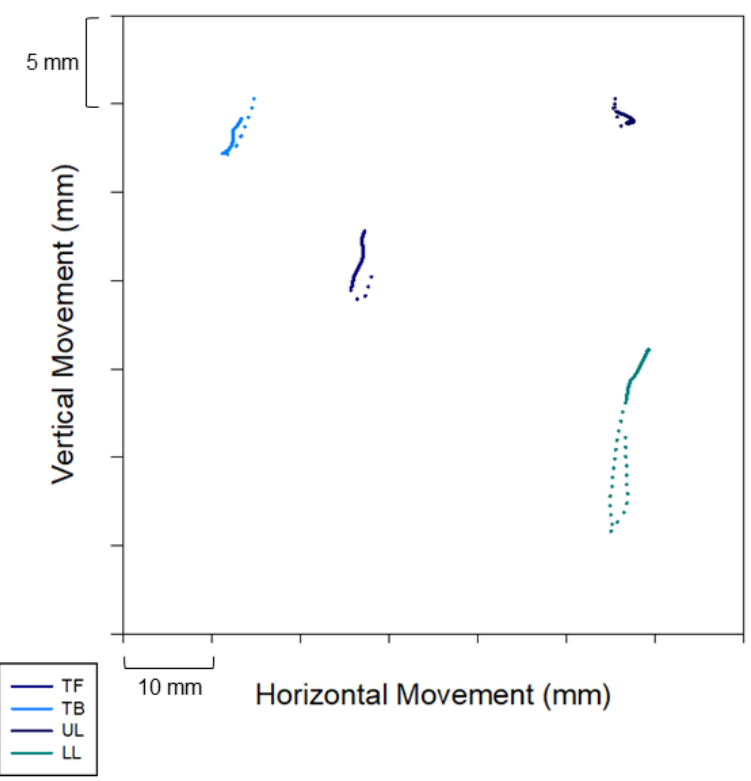

Figure 5. Comparison of the UL, LL, TF, and TB articulators during a conversational production of "buy." The solid lines indicate the articulatory movement made during the stop closure. The dotted lines indicate the movement during the following diphthong

Speaking mode changes. Analyzing the effects of speaking mode changes on the articulatory distances yielded interesting results. We found that when the $\mathrm{HC}$ group produced more clear speech, their LL movement distances were decreased relative to their movement with a conversational speaking mode. Conversely, when the PD group produced more clear speech, their LL movement distance was increased relative to their movement with a conversational speaking mode.

To help explain these tendencies, we examined the UL and LL movement distances during the initial- and final-movement of the closure duration. The initial closure movement was defined as the interval from the closure onset (i.e., peak vertical velocity of the LL) to the peak lip aperture (i.e., the moment in which the distance between the UL and LL sensors is at its most minimum value). The final closure movement was defined as the interval between the peak lip aperture and the closure offset (see Figure 6). 
$\mathrm{HC} 2$

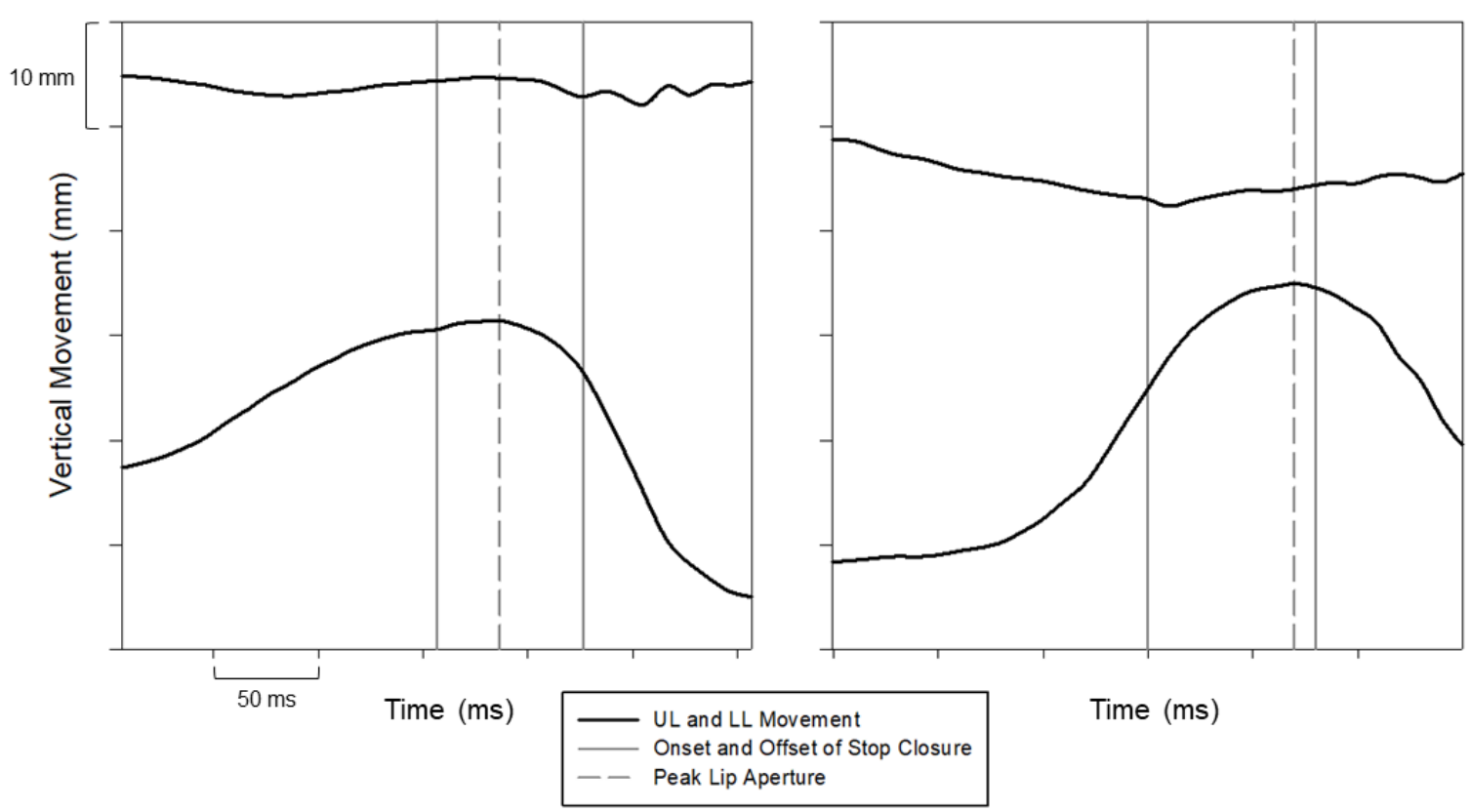

Figure 6. Comparison of UL and LL closure onset and offset movement of consonant /b/ produced by speakers HC 2 and PD 2. The first and third vertical lines indicate the onset and offset of the stop closure. The second vertical line indicates the moment of peak lip aperture. UL = upper lip; $\mathrm{LL}=$ lower lip; $\mathrm{HC}=$ healthy control; $\mathrm{PD}=$ Parkinson's disease.

A closer examination of the initial- and final-labial movement distances during the closure phase of more clear speech revealed the PD group demonstrated greater initial-labial movement $(M=3.51 \mathrm{~mm})$ compared to the $\mathrm{HC}$ group $(M=2.73 \mathrm{~mm})$ as seen in Figure 6 . After the closure onset, the PD group continues to raise their LL until the point of peak lip aperture. At that moment, they reverse their LL movement to release the intraoral pressure and produce the stop burst. The HC group demonstrates a similar pattern. However, after the closure onset, they do not continue to raise the LL to the same magnitude as the PD group. We hypothesize this observation of greater LL initial-movement within the PD group suggests that there are motoric differences between the PD and HC groups when creating a labial seal. The HC group's lip contact force may be greater than the PD group's lip contact force. This would explain why the PD group appears to compensate for reduced lip contact pressure by producing greater LL initial- 
movement closure during the closure phase. Perhaps, this greater LL initial-movement helps create the air-tight labial seal needed to achieve the intraoral pressure build-up required for stop bursts.

Assuming this is true, this would explain the differences in labial movement distances between the two groups when producing more clear speech. As previously mentioned, clear speaking modes are associated with greater intensity (increased dB SPL) relative to conversational speaking modes (Tjaden \& Martel-Sauvageau, 2017). Therefore, we can assume both groups were attempting to produce greater intraoral pressure during the stop closure for the clear speaking mode which would require a tighter labial seal. This explains why the HC group demonstrate reduced LL closure initial-movement (i.e., they have adequate lip contact force to create the air-tight seal), whereas the PD group demonstrate greater LL closure initial-movement (i.e., they lack the adequate lip contact force and instead compensate with exaggerated LL and jaw movement to create the air-tight seal). Additionally, our findings of reduced vertical UL movement distances produced by the PD group support the idea of insufficient lip contact force. However, without a direct measure of labial contact force, our hypothesis cannot be tested at this time.

For speaking mode changes from conversational to less clear, the HC group decreased their LL movement averages from $9.22 \mathrm{~mm}$ to $6.93 \mathrm{~mm}$. Interestingly, the opposite trend was observed in the PD group. From conversational to less clear speaking modes, the PD group increased their LL movement averages from $6.04 \mathrm{~mm}$ to $6.60 \mathrm{~mm}$.

Changes in speaking mode appear to have little to no effect on the TF or TB movement distances during the stop closure interval. Every speaker (except HC 2) produced very similar average movement distances across speaking modes. Relative to conversational speech, 
Participant HC 2 produced reduced TF and TB movement distances during more clear and less clear speaking conditions. It remains unclear why these idiosyncratic movement patterns were present.

\section{Displacement}

Displacement was calculated as the distance between the onset and offset of the stop closure duration. Like the measures for distance, the displacement data was normalized with respect to the stop closure duration. Data normalization was completed to ensure that speakers with longer closure durations were not misinterpreted as having greater displacement measures.

Healthy speakers vs. speakers with PD. Comparisons of average labial displacement (combined horizontal and vertical displacement) measures did not show any clear differences between the two groups. However, examining the average vertical displacement alone revealed the $\mathrm{HC}$ group produced greater vertical displacement values $(M=1.59 \mathrm{~mm}$ for $\mathrm{UL} ; M=5.77$ $\mathrm{mm}$ for $\mathrm{LL}$ ) than the PD group ( $M=-0.07 \mathrm{~mm}$ for $\mathrm{UL} ; M=0.85 \mathrm{~mm}$ for $\mathrm{LL}$ ). In other words, for the HC group, the UL and LL position tended to be more vertically displaced at the closure offset than their position at the closure onset (see Figure 7). The same trend was seen in the PD group. However, the UL and LL were less displaced compared to the HC group.

An examination of the lingual displacement (combined horizontal and vertical displacement) revealed similar results. Except for PD 1, the HC group demonstrated greater lingual displacement $(M=9.01 \mathrm{~mm}$ for TF; $M=6.89 \mathrm{~mm}$ for TB) than the PD group $(M=5.77$ $\mathrm{mm}$ for TF; $M=5.97 \mathrm{~mm}$ for TB). As previously mentioned, the speech of PD 1 was characterized as the most severe of all of the participants. We believe this speaker is attempting to compensate for their speech severity by producing exaggerated lingual movement. 
$\mathrm{HC} 2$

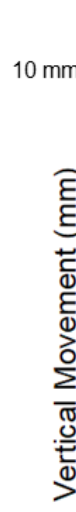

$0 \mathrm{~mm}$

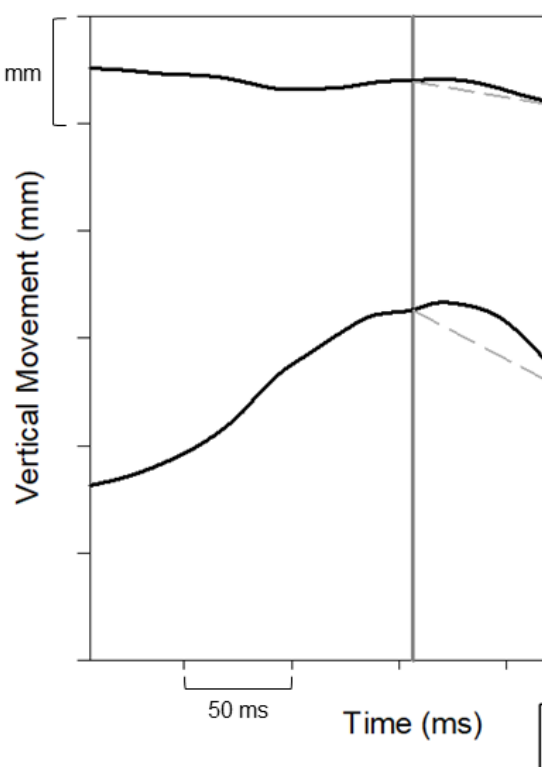

PD 3

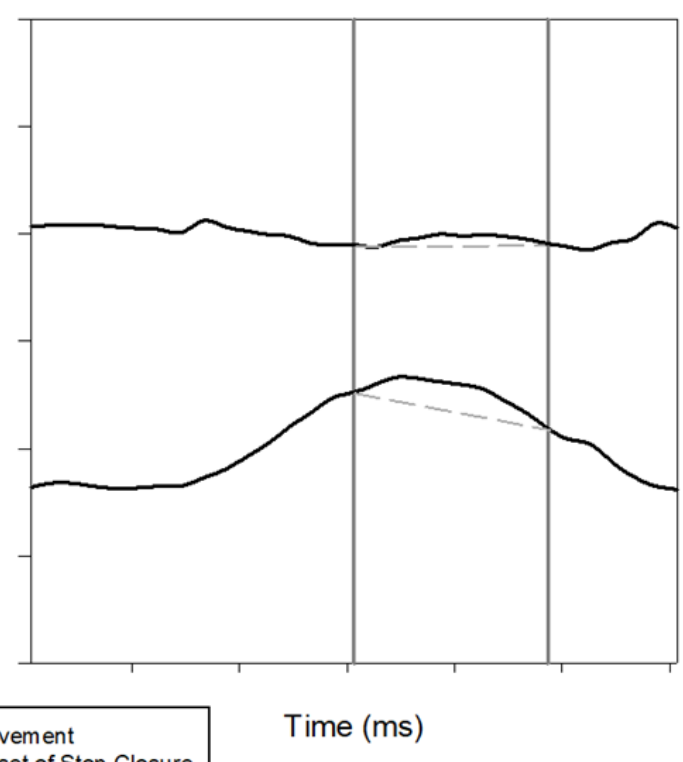

Onset and Offset of Stop Closure Displacement

Figure 7. Comparison of UL and LL displacement during the stop closure of consonant /b/ produced by speakers HC 2 and PD 3. The two vertical lines indicate the onset and offset of the stop closure. The dotted line between the onset and offset represents the displacement. UL $=$ upper lip; LL = lower lip; HC = healthy control; PD = Parkinson's disease.

Speaking mode changes. It appears the two speaking groups made similar UL articulatory modifications across speaking modes. All speakers producing more clear speech tended to displace their UL to a lesser degree than UL displacement during conversational speech. The same trend was seen for less clear speaking modes, however, to a greater magnitude (i.e., the UL was least displaced within less clear speaking modes).

For speakers producing more clear speech, the LL tended to be more displaced than in conversational speaking modes. In less clear speaking conditions, the HC speakers (and PD 2) produced less displaced movement $(M=4.74 \mathrm{~mm})$ compared to conversational speaking conditions $(M=7.26 \mathrm{~mm})$. Similar to our findings of unexpected LL distance changes from conversational to less clear speaking conditions, the PD speakers produced more displaced LL 
movement in less clear speaking conditions $(M=3.69 \mathrm{~mm})$ compared to conversational speaking conditions $(M=3.56 \mathrm{~mm})$.

\section{Speed}

The speed of articulatory movement during the stop closure was calculated as the distance of articulatory movement as a function of time. The speed of labial movement for both speaking groups appears to be comparable (except PD 4). Participant PD 4 demonstrated uniquely slow labial movement. The speed of lingual movement was found to be quicker in the HC group (and PD 1) than the PD group. Consistent with our findings of distance and displacement, speaker PD 1 demonstrated quicker articulatory speeds potentially as a compensatory strategy. Furthermore, speaking mode changes did not result in any noteworthy changes to the speed of labial or lingual movement for either speaking group.

\section{Reliability}

Two participants, one healthy speaker and one speaker with PD (33\%), were randomly selected for the reliability measures. Intrameasurer reliability was established by re-segmenting the acoustic signal to identify the $200 \mathrm{~ms}$ prior to the stop burst. The total difference in duration between the first and second measures was $15.51 \mathrm{~ms}$. A pairwise, Pearson product moment correlation between the two sets of measures was 1.00. 
Table 3. Articulatory Measurements for Each Articulator Produced by Participants Using a Conversational Speaking Mode

\begin{tabular}{|c|c|c|c|c|c|c|c|c|}
\hline \multirow{2}{*}{ Participant } & \multirow{2}{*}{$\begin{array}{l}\text { Duration } \\
(\mathrm{ms})\end{array}$} & \multicolumn{3}{|c|}{ Distance (mm) } & \multicolumn{3}{|c|}{ Displacement (mm) } & \multirow{2}{*}{$\begin{array}{c}\text { Speed } \\
(\mathrm{mm} / \mathrm{ms})\end{array}$} \\
\hline & & $\mathrm{X}$ & $\mathrm{Y}$ & $\mathrm{XY}$ & $\mathrm{X}$ & $\mathrm{Y}$ & $\mathrm{XY}$ & \\
\hline \multicolumn{9}{|c|}{ Tongue Front } \\
\hline $\mathrm{HC} 1$ & 64 & 9.8007 & 6.3344 & 13.5768 & -6.6735 & 5.9847 & 9.1893 & 0.1358 \\
\hline HC 2 & 60 & 2.1774 & 8.5207 & 8.9024 & 2.1437 & 8.5207 & 8.8323 & 0.0890 \\
\hline PD 1 & 128 & 2.9164 & 10.8713 & 11.4872 & 2.1579 & 10.7481 & 11.0109 & 0.1149 \\
\hline PD 2 & 78 & 2.7132 & 2.6549 & 4.0491 & -1.9242 & 1.6763 & 3.1465 & 0.0405 \\
\hline PD 3 & 83 & 2.3195 & 4.0459 & 4.8614 & 2.0434 & 3.8298 & 4.3668 & 0.0469 \\
\hline $\operatorname{PD} 4^{\mathrm{a}}$ & 82 & - & - & - & - & - & - & - \\
\hline \multicolumn{9}{|c|}{ Tongue Back } \\
\hline $\mathrm{HC} 1$ & 64 & 4.2551 & 6.4758 & 8.4204 & -2.5883 & 6.0376 & 6.7931 & 0.0842 \\
\hline $\mathrm{HC} 2$ & 60 & 2.3470 & 6.6535 & 7.1667 & 1.7576 & 6.6535 & 6.9801 & 0.0717 \\
\hline PD 1 & 128 & 3.2695 & 10.1978 & 10.8543 & 2.5785 & 9.9467 & 10.3038 & 0.1085 \\
\hline PD 2 & 78 & 2.4874 & 2.7015 & 3.8401 & -2.4485 & 1.0216 & 3.3960 & 0.0384 \\
\hline PD 3 & 83 & 2.7701 & 3.4621 & 4.7405 & 2.5504 & 3.4621 & 4.3446 & 0.0442 \\
\hline $\operatorname{PD} 4^{\mathrm{a}}$ & 82 & - & - & - & - & - & - & - \\
\hline \multicolumn{9}{|c|}{ Upper Lip } \\
\hline $\mathrm{HC} 1$ & 64 & 2.0832 & 2.2509 & 3.4689 & -0.5622 & 1.3625 & 1.7264 & 0.0347 \\
\hline $\mathrm{HC} 2$ & 60 & 1.5630 & 1.8849 & 2.6089 & 1.4458 & 1.8082 & 2.3685 & 0.0261 \\
\hline PD 1 & 128 & 1.6660 & 1.3370 & 2.4052 & 0.8725 & 0.7388 & 1.3360 & 0.0241 \\
\hline PD 2 & 78 & 2.1377 & 1.0131 & 2.5098 & -1.9351 & -0.0864 & 2.0555 & 0.0251 \\
\hline PD 3 & 83 & 3.1560 & 1.7664 & 3.8625 & 1.7164 & -0.5707 & 1.8263 & 0.0386 \\
\hline PD 4 & 82 & 0.6084 & 0.6564 & 0.9987 & -0.4082 & -0.3791 & 0.6519 & 0.0100 \\
\hline \multicolumn{9}{|c|}{ Lower Lip } \\
\hline $\mathrm{HC} 1$ & 64 & 5.8509 & 6.1173 & 8.7582 & -3.9924 & 3.6562 & 5.4659 & 0.0876 \\
\hline $\mathrm{HC} 2$ & 60 & 4.5620 & 8.2658 & 9.6757 & 4.4330 & 7.8830 & 9.0480 & 0.0968 \\
\hline PD 1 & 128 & 3.0929 & 6.9360 & 7.7638 & 2.1296 & 1.9789 & 3.2419 & 0.0776 \\
\hline PD 2 & 78 & 1.9305 & 7.3154 & 7.7795 & -0.0536 & -2.9608 & 5.5250 & 0.0778 \\
\hline PD 3 & 83 & 3.9257 & 4.5834 & 6.5493 & 2.5223 & 3.2661 & 4.1453 & 0.0648 \\
\hline PD 4 & 82 & 1.1008 & 1.6190 & 2.0478 & 0.7185 & 1.1218 & 1.3389 & 0.0205 \\
\hline
\end{tabular}

Note. $\mathrm{HC}=$ healthy control; $\mathrm{PD}=$ Parkinson's disease

${ }^{a}$ Lingual movement data for speaker PD 4 was excluded due to sensor attachment issues. 
Table 4. Articulatory Measurements for Each Articulator Produced by Participants Using a More Clear Speaking Mode

\begin{tabular}{|c|c|c|c|c|c|c|c|c|}
\hline \multirow{2}{*}{ Participant } & \multirow{2}{*}{$\begin{array}{l}\text { Duration } \\
\quad(\mathrm{ms})\end{array}$} & \multicolumn{3}{|c|}{ Distance (mm) } & \multicolumn{3}{|c|}{ Displacement (mm) } & \multirow{2}{*}{$\begin{array}{c}\text { Speed } \\
(\mathrm{mm} / \mathrm{ms})\end{array}$} \\
\hline & & $\mathrm{X}$ & $\mathrm{Y}$ & $\mathrm{XY}$ & $\mathrm{X}$ & $\mathrm{Y}$ & $\mathrm{XY}$ & \\
\hline \multicolumn{9}{|c|}{ Tongue Front } \\
\hline $\mathrm{HC} 1$ & 60 & 12.2126 & 5.6382 & 14.3427 & -2.1537 & 5.3605 & 6.8615 & 0.1434 \\
\hline HC 2 & 76 & 0.9299 & 7.0194 & 7.1812 & 0.3577 & 6.9592 & 6.9879 & 0.0718 \\
\hline PD 1 & 124 & 3.1735 & 11.3637 & 11.9970 & 2.5158 & 11.0041 & 11.3596 & 0.1200 \\
\hline PD 2 & 58 & 2.3602 & 2.4691 & 3.8592 & -0.1297 & 1.3986 & 2.0749 & 0.0386 \\
\hline PD 3 & 76 & 2.7579 & 4.9765 & 5.9968 & 1.8492 & 4.5626 & 4.9522 & 0.0600 \\
\hline $\mathrm{PD} 4^{\mathrm{a}}$ & 78 & - & - & - & - & - & - & - \\
\hline \multicolumn{9}{|c|}{ Tongue Back } \\
\hline $\mathrm{HC} 1$ & 60 & 4.0732 & 6.7446 & 8.6974 & -2.3550 & 6.7446 & 7.4774 & 0.0870 \\
\hline HC 2 & 76 & 0.7667 & 5.2362 & 5.4376 & -0.1972 & 5.2362 & 5.2971 & 0.0544 \\
\hline PD 1 & 124 & 3.5546 & 10.9891 & 11.7252 & 2.8896 & 10.5706 & 11.0370 & 0.1173 \\
\hline PD 2 & 58 & 2.4627 & 3.1667 & 4.3303 & -0.8801 & 1.4844 & 2.9809 & 0.0433 \\
\hline PD 3 & 76 & 2.3269 & 3.9920 & 4.8598 & 2.0720 & 3.9409 & 4.4621 & 0.0486 \\
\hline $\operatorname{PD} 4^{\mathrm{a}}$ & 78 & - & - & - & - & - & - & - \\
\hline \multicolumn{9}{|c|}{ Upper Lip } \\
\hline $\mathrm{HC} 1$ & 60 & 1.7951 & 2.9954 & 3.6356 & -0.0337 & 1.2952 & 1.6768 & 0.0364 \\
\hline HC 2 & 76 & 1.1279 & 2.3304 & 2.7059 & 0.7742 & 1.9048 & 2.0769 & 0.0271 \\
\hline PD 1 & 124 & 2.0508 & 1.8417 & 3.0526 & 1.0821 & 0.2321 & 1.5487 & 0.0305 \\
\hline PD 2 & 58 & 1.7693 & 1.9847 & 3.0463 & -1.4054 & -1.2663 & 2.2117 & 0.0305 \\
\hline PD 3 & 76 & 2.3625 & 1.5836 & 3.1052 & 1.5434 & -0.1067 & 1.6187 & 0.0311 \\
\hline PD 4 & 78 & 0.6772 & 0.4096 & 0.8582 & 0.4755 & -0.1378 & 0.5255 & 0.0086 \\
\hline \multicolumn{9}{|c|}{ Lower Lip } \\
\hline $\mathrm{HC} 1$ & 60 & 4.9171 & 5.9895 & 8.3047 & -3.5639 & 5.0228 & 6.2366 & 0.0830 \\
\hline HC 2 & 76 & 3.2824 & 6.9356 & 7.7763 & 2.8648 & 5.7407 & 6.4248 & 0.0778 \\
\hline PD 1 & 124 & 2.7923 & 7.0592 & 7.8418 & 1.9350 & 3.6781 & 4.5662 & 0.0784 \\
\hline PD 2 & 58 & 2.0698 & 7.8192 & 8.1799 & 0.7812 & -2.9326 & 6.8373 & 0.0818 \\
\hline PD 3 & 76 & 3.6235 & 5.2118 & 6.9737 & 1.9136 & 3.8070 & 4.3075 & 0.0697 \\
\hline PD 4 & 78 & 1.3644 & 2.0141 & 2.5070 & 1.1552 & 1.8119 & 2.1524 & 0.0251 \\
\hline
\end{tabular}

Note. $\mathrm{HC}=$ healthy control; $\mathrm{PD}=$ Parkinson's disease

${ }^{a}$ Lingual movement data for speaker PD 4 was excluded due to sensor attachment issues. 
Table 5. Articulatory Measurements for Each Articulator Produced by Participants Using a Less Clear Speaking Mode

\begin{tabular}{|c|c|c|c|c|c|c|c|c|}
\hline \multirow{2}{*}{ Participant } & \multirow{2}{*}{$\begin{array}{l}\text { Duration } \\
(\mathrm{ms})\end{array}$} & \multicolumn{3}{|c|}{ Distance $(\mathrm{mm})$} & \multicolumn{3}{|c|}{ Displacement (mm) } & \multirow{2}{*}{$\begin{array}{c}\text { Speed } \\
(\mathrm{mm} / \mathrm{ms})\end{array}$} \\
\hline & & $X$ & $\mathrm{Y}$ & $\mathrm{XY}$ & $\mathrm{X}$ & $\mathrm{Y}$ & $\mathrm{XY}$ & \\
\hline \multicolumn{9}{|c|}{ Tongue Front } \\
\hline $\mathrm{HC} 1$ & 83 & 12.7777 & 6.1714 & 15.2586 & -4.6859 & 5.3099 & 7.2280 & 0.1526 \\
\hline $\mathrm{HC} 2$ & 58 & 0.8542 & 3.5917 & 3.7537 & 0.6132 & 3.5917 & 3.6658 & 0.0375 \\
\hline PD 1 & 154 & 2.3991 & 8.5528 & 8.9846 & 2.0594 & 8.2294 & 8.4886 & 0.0898 \\
\hline PD 2 & 102 & 2.1230 & 3.1912 & 4.0479 & -1.6599 & 2.8154 & 3.3673 & 0.0405 \\
\hline PD 3 & 72 & 1.7342 & 3.1845 & 3.7919 & 1.0689 & 3.0951 & 3.2977 & 0.0379 \\
\hline $\mathrm{PD} 4^{\mathrm{a}}$ & 96 & - & - & - & - & - & - & - \\
\hline \multicolumn{9}{|c|}{ Tongue Back } \\
\hline $\mathrm{HC} 1$ & 83 & 4.3295 & 5.3613 & 7.7666 & -1.6460 & 4.5855 & 5.0858 & 0.0777 \\
\hline $\mathrm{HC} 2$ & 58 & 0.5858 & 2.9568 & 3.0461 & 0.0391 & 2.9568 & 2.9608 & 0.0305 \\
\hline PD 1 & 154 & 2.8131 & 8.4895 & 9.0548 & 2.3835 & 8.1719 & 8.5179 & 0.0905 \\
\hline PD 2 & 102 & 2.7433 & 3.5775 & 4.9447 & -2.2497 & 2.7991 & 3.8041 & 0.0494 \\
\hline PD 3 & 72 & 2.0127 & 2.3771 & 3.3940 & 1.0261 & 2.2763 & 2.5992 & 0.0339 \\
\hline $\mathrm{PD} 4^{\mathrm{a}}$ & 96 & - & - & - & - & - & - & - \\
\hline \multicolumn{9}{|c|}{ Upper Lip } \\
\hline $\mathrm{HC} 1$ & 83 & 1.9313 & 2.9447 & 3.9751 & 0.3219 & 1.3575 & 1.5842 & 0.0398 \\
\hline HC 2 & 58 & 1.4115 & 1.2781 & 1.9839 & 1.1149 & 1.1034 & 1.7805 & 0.0198 \\
\hline PD 1 & 154 & 1.9337 & 1.3243 & 2.5736 & 1.0402 & 0.6326 & 1.2427 & 0.0257 \\
\hline PD 2 & 102 & 2.2284 & 1.5311 & 2.9574 & -2.0280 & -0.3775 & 2.2499 & 0.0296 \\
\hline PD 3 & 72 & 3.8053 & 2.4125 & 4.6925 & 0.8654 & -0.6113 & 1.3994 & 0.0469 \\
\hline PD 4 & 96 & 0.4014 & 0.5586 & 0.7616 & 0.2322 & -0.2204 & 0.3838 & 0.0076 \\
\hline \multicolumn{9}{|c|}{ Lower Lip } \\
\hline $\mathrm{HC} 1$ & 83 & 4.5538 & 5.8638 & 7.7452 & -1.4503 & 1.7175 & 3.3965 & 0.0775 \\
\hline $\mathrm{HC} 2$ & 58 & 3.2666 & 5.0905 & 6.1115 & 3.2605 & 5.0905 & 6.0855 & 0.0611 \\
\hline PD 1 & 154 & 2.8069 & 6.8900 & 7.5570 & 1.7734 & 2.1936 & 3.5936 & 0.0756 \\
\hline PD 2 & 102 & 2.8742 & 8.3098 & 8.9302 & -1.3807 & 2.1978 & 4.1329 & 0.0893 \\
\hline PD 3 & 72 & 3.8353 & 5.4493 & 7.2392 & 2.4949 & 4.1470 & 4.9172 & 0.0724 \\
\hline PD 4 & 96 & 1.5374 & 2.0813 & 2.6824 & 1.2868 & 1.6600 & 2.1088 & 0.0268 \\
\hline
\end{tabular}

Note. $\mathrm{HC}=$ healthy control; $\mathrm{PD}=$ Parkinson's disease

${ }^{a}$ Lingual movement data for speaker PD 4 was excluded due to sensor attachment issues. 


\section{Discussion}

The current study was an exploratory study with the purpose of investigating the articulatory kinematics of the stop closure interval of bilabial stop consonants produced by speakers with PD. Very few studies have investigated the articulatory movements during the stop closure (see Löfqvist \& Gracco, 1997), and to our knowledge, no previous studies have investigated the articulatory movements during the stop closure among speakers with PD.

We were interested in the stop closure for several reasons. Firstly, stop consonants comprise $29 \%$ of all consonants and are likely to play an important role in the speech intelligibility of speakers with PD (Kent \& Kim, 2003). Secondly, speakers with PD have been reported to have abnormal speech characteristics during the stop closure, such as spirantization, or the presence of multiple stop bursts (for spirantization, see Ramig, Titze, Scherer, \& Ringel, 1988; Weismer, Yunusova, \& Bunton, 2012; for multiple stop bursts, see Parveen \& Goberman, 2014). Finally, due to the silent nature of the closure phase, limited perceptual and acoustic information has been reported during this time interval. Therefore, a kinematic approach was used due to its ability to examine articulatory events when little-to-no acoustic signals are available.

Our study sought to answer two research questions: (1) how do articulatory movements during the closure phase of bilabial stop consonant $/ b /$ differ with respect to distance, displacement, duration, and speed of motion between the PD and HC groups; and (2) do speakers with PD exhibit changes in articulatory movements when they voluntarily vary the degree of speech intelligibility? If so, are the articulatory changes similar to the changes made by the $\mathrm{HC}$ group? 


\section{Healthy Speakers vs. Speakers with PD}

Our results suggest there are differences in articulatory movements during the closure phase of bilabial stop consonant between the HC and PD groups. Specifically, with respect to stop closure duration, we found the PD group produced longer closure durations compared to the HC group. Previous investigations of stop closure have reported closure durations for bilabial stop consonants ranging from 55 to 92 ms (Crystal \& House, 1988; Stathopoulous \& Weismer, 1983). Our findings were comparable to these findings.

Examination of the articulatory distance differences between the two groups revealed the PD group produced reduced UL movement and reduced LL movement compared to the HC group. Specifically, the HC group produced greater vertical UL movement and greater horizontal LL movement compared to the PD group. We found comparable LL vertical movement distances between the two groups, suggesting the PD group may be able to compensate for articulatory changes with increased LL and jaw movement. Similar findings were observed in lingual movement. The HC group demonstrated greater TF and TB movement distances than the PD group.

Consistent with our findings of group differences for distance measures, we found measures of displacement reduced in the PD group relative to the HC group. The HC group demonstrated greater vertical displacement of the UL and LL. These findings are in agreement with previous studies reporting reduced labial displacement among PD speakers (e.g., Caligiuri, 1987). Concerning lingual movement, we found increased lingual displacement among the HC group compared to the PD group. 


\section{Speaking Mode Changes}

Regarding our second research question, we found that the two speaking groups made observable, but different, articulatory changes during the closure interval when modifying speaking mode. When analyzing the effects of speaking modes on stop closure duration, we hypothesized more clear speaking conditions would elicit longer closure durations and less clear speaking conditions would elicit smaller closure durations. This hypothesis was based on previous reports of segment duration lengthening for vowels with clear speaking conditions produced by speakers with PD (Tjaden, Lam, \& Wilding, 2013). Interestingly, the opposite was observed: relative to a conversational speaking mode, every speaker (except HC 2) tended to decrease the stop closure duration during more clear speaking conditions, and increase the stop closure duration during less clear speaking conditions. The same trend was observed with VOT.

Upon investigation of the following sound (i.e., diphthong /aI/ of "buy"), we found vowel durations consistent with those reported by Tjaden et al. (2013). For more clear speech, the vowel duration lengthened, and for less clear speech, the vowel duration shortened. These findings suggest that when a speaker attempts to produce more clear speech, they shorten the duration of the stop consonant to allow more time to produce the following vowel.

The distance and displacement trends produced by the HC group are what we expected to see (i.e., more LL displacement for more clear speech and less LL displacement for less clear speech). However, the PD group demonstrated unusual distance and displacement tendencies when modifying their speaking modes. When producing more clear speech, the PD group produced greater distance measures relative to the conversational speaking mode. Further investigation of the initial- and final-closure movement revealed the PD group consistently produced greater initial-closure movement compared to the HC group. It was hypothesized the 
greater initial-closure movement was a result of the PD group's attempt to compensate for a reduced lip contact pressure needed to create a complete labial seal (and thus avoiding spirantization). In the current study, no observations of spirantization were observed. However, this may be due to the PD group's ability to compensate for inadequate labial closure by producing exaggerated lower lip and jaw movement. This finding is consistent with previous reports of more frequent observations of spirantization in alveolar and velar stops compared to bilabial stops (Logemann \& Fisher, 1981).

Unlike the HC group, the PD group increased their distance and displacement values when producing less clear speech. Therefore, it appears that when PD speakers are asked to produce more clear speech, they can achieve this by exaggerating their articulatory movements (i.e., exaggerated lip and jaw excursions). However, when the PD speakers are asked to produce more nuanced and subtle movement changes (i.e., less clear speaking conditions), the PD speakers lack the articulatory precision needed to coordinate reduced labial movement.

In summary, we found that speakers with $\mathrm{PD}$ and $\mathrm{HC}$ demonstrate unique articulatory movement patterns during the closure interval of bilabial stop consonant production. The effects of speaking mode modifications via direct magnitude estimation revealed speakers with PD could successfully produce more clear speech and less clear speech. However, there appear to be group differences in the approach used to achieve the desired speaking mode.

\section{Clinical Implications}

As previously mentioned, speaking mode modifications among speakers with dysarthria (i.e., loud speech, clear speech, slow speech) are investigated in an effort to establish a theoretical foundation for the development of behavioral treatment methods. In the present study, we investigated two speaking conditions: more clear and less clear. Tjaden et al. (2014) found 
clear speaking modes elicit greater measures of speech intelligibility (i.e., F2 slopes) than loud or slow speaking conditions. However, before the current study, it was unknown how these speaking mode modifications affect the articulatory quality of speech segments (e.g., stop consonants).

In the current study, we found that more clear speech successfully elicits greater articulatory movement (both articulatory distances and displacement). These reports support the efficacy of using more clear speaking modes in a clinical context to elicit better speech among speakers with PD. From the findings of the current study, it appears speakers with PD may achieve a more clear speaking mode by producing exaggerated jaw and lip movement. However, it should be noted that when speakers with PD were asked to produce smaller, more nuanced, articulatory movements (i.e., less clear), they were unable to achieve the desired effects. With respect to these findings, clinicians should be cognizant of PD speakers' difficulty producing smaller articulatory changes, and instead, focus on more achievable articulatory modifications (e.g., increased jaw excursions for more clear speaking modes).

\section{Limitations}

Several limitations should be noted for this study. First, the number of participants was relatively low, and therefore their data may not be representative of the entire population. Future studies should include more participants. Secondly, the current study did not inspect the articulatory movement of healthy aging females despite having a female participant in the PD group. Additionally, Löfqvist and Gracco (1997) reported the peak vertical velocity of the LL consistently occurred at the onset of the stop closure. Therefore, within the present study, the peak vertical velocity of the LL was used to identify the closure onset. However, without a direct measure of intraoral pressure, we cannot test the accuracy of using LL peak vertical velocity for 
the identification of closure onset. Finally, our findings of increased LL movement distances among speakers with PD was theorized to be caused by compensatory LL and jaw movements due to reduced lip contact force during the stop closure. However, without measures of lip contact force, we cannot test this hypothesis.

\section{Future Research}

In the present study, the lingual and labial movement were not decoupled from the jaw. Jaw-decoupling would allow for the examination of lingual and LL movement without the effects of jaw movements. In the future, we plan to apply the jaw-decoupling procedure outlined by Westbury, Lindstrom, and McClean (2002). Additionally, the current study examined the articulatory movement of bilabial stops in a CV structure. In future studies, we hope to complete the articulatory profile for $\mathrm{CV}$ structures, including alveolar and velar stop consonants. Finally, this study explored the effects of more clear and less clear speaking modes. Future research should continue to investigate the articulatory effects of more clear speaking modes at a segmental level. 


\section{Conclusion}

The purpose of the current study was to investigate the articulatory kinematics during the stop closure interval of bilabial stop consonants produced by speakers with PD and healthy speakers. Also, we investigated the effects of speaking mode modifications (i.e., conversational, more clear, and less clear speaking modes) on articulatory kinematics in speakers with PD and healthy speakers. Consistent with previous perceptual and acoustic findings, we found speakers with PD produce reduced articulatory movement compared to the healthy speakers. Additionally, it was observed that speakers with PD produced exaggerated LL and jaw movements to achieve complete labial closure and to avoid spirantization. Despite these movement differences, both speaking groups successfully modified their speech movements to achieve perceptually more clear speaking conditions. 


\section{References}

Bradlow, A., Torretta, G., \& Pisoni, D. (1996). Intelligibility of normal speech I: Global and fine-grained acoustic-phonetic talker characteristics,. Speech Communication, 20(3), 255272.

Bunton, K., \& Weismer, G. (2001). The relationship between perception and acoustics for a high-low vowel contrast produced by speakers with dysarthria. Journal of Speech, Language, and Hearing Research, 44(6), 1215-1228.

Caligiuri, M. (1987). Labial kinematics during speech in patients with Parkinsonian rigidity. Brain, 110(4), 1033-1044.

Canter, G. (1963). Speech characteristics of patients with Parkinson's disease: 1. Intensity, pitch, and duration. Journal of Speech and Hearing Disorders, 28(3), 221-229.

Crystal, T., \& House, A. (1988). The duration of American-English stop consonants: An overview. Journal of Phonetics, 16(2), 285-294.

Cushine-Sparrow, D., Adams, S., Knowles, T., Leszcz, T., \& Jog, M. (2016). Effects of multitalker noise on the acoustics of stop consonants in Parkinson's disease. Western Papers in Linguistics / Cahiers linguistiques de Western, 3(2), Article 2.

Darley, F., Aronson, A., \& Brown, J. (1969a). Differential diagnostic patterns of dysarthria. Journal of Speech, Language, and Hearing Research, 12(2), 246-269.

Darley, F., Aronson, A., \& Brown, J. (1969b). Clusters of deviant speech dimensions in the dysarthrias. Journal of Speech, Language, and Hearing Research, 12(3), 462-496.

Darling, M., \& Huber, J. (2011). Changes to articulatory kinematics in response to loudness cues in individuals with Parkinson's disease. Journal of Speech, Language, and Hearing Research, 54, 1247-1259.

Dorland, W. (1965). Dorland's Illustrated Medical Dictionary (24 ed.). Philadelphia \& London: W. B. Saunders.

Dromey, C., Jang, G., \& Hollis, K. (2013). Assessing correlations between lingual movements and formants. Speech Communication, 55, 315-328.

Duffy, J. (2013). Motor speech disorders: Substrates, differential diagnosis, and management. Rochester, Minnesota: Elselvier Mosby.

Fischer, E., \& Goberman, A. (2010). Voice onset time in Parkinson disease. Journal of Communication Disorders, 43, 21-34.

Flint, A., Black, S., Cambell-Taylor, I., Gailey, G., \& Levinton, C. (1992). Acoustic analysis in the differentiation between Parkinson's disease and major depression. Journal of Psycholinguistic Research, 21, 383-399. 
Forrest, K., \& Weismer, G. (1995). Dynamic aspects of lower lip movement in Parkinsonian and neurologically normal geriatric speakers' production of stress. Journal of Speech, Language, and Hearing Research, 38, 260-272.

Forrest, K., Weismer, G., \& Turner, G. (1989). Kinematic, acoustic, and perceptual analyses of connected speech produced by Parkinsonian and normal geriatric males. Journal of the Acoustical Society of America, 85(6), 2608-2622.

Goberman, A., \& Elmer, L. (2005). Acoustic analysis of clear versus conversational speech in individuals with Parkinson's disease. Journal of Communication Disorders, 38(3), 215 230.

Hirsch, L., Jette, N., Frolkis, A., Steeves, T., \& Pringsheim, T. (2016). The incidence of parkinson's disease: A systematic review and meta-analysis. Neuroepidemiology, 46, 292-300.

Hochstadt, J., Nakano, H., Lieberman, P., \& Friedman, J. (2005). The roles of sequencing and verbal working memory in sentence comprehension deficits in Parkinson's disease. Brain and Language, 97(3), 243-257.

Jang, H., Boltz, D., Webster, R., \& Smeyne, R. (2008). Viral parkinsonism. Biochimica et Biophysica Acta - Molecular Basis of Disease, 1792(7), 714-721.

Kent, R., \& Kim, Y. (2003). Toward an acoustic typology of motor speech disorders. Clinical Linguistics \& Phonetics, 17(6), 427-445.

Kent, R., \& Rosenbek, J. (1982). Prosodic disturbance and neurologic lesion. Brain and Language, 15, 259-291.

Kim, Y., Kent, R., \& Weismer, G. (2011). An acoustic study of the relationships among neurologic disease, dysarthria type, and severity of dysarthria. Journal of Speech, Language, and Hearing Research, 54, 417-430.

Kim, Y., Weismer, G., Kent, R., \& Duffy, J. (2009). Statistical models of F2 slope in relation to severity of dysarthria. Folia Phoniatrica et Logopaedica, 61, 329-335.

Kleinow, J., Smith, A., \& Ramig, L. (2001). Speech motor stability in IPD: Effects of rate and loudness manipulations. Journal of Speech, Language, and Hearing Research, 44, 10411051.

Löfqvist, A., \& Gracco, V. (1997). Lip and jaw kinematics in bilabial stop consonant production. Journal of Speech, Language, and Hearing Research, 40, 877-893.

Löfqvist, A., \& Gracco, V. (2002). Control of oral closure in lingual stop consonant production. Journal of the Acoustical Society of America, 111, 2811-2827.

Lansford, K., \& Liss, J. (2014). Vowel acoustics in dysarthria: Speech disorder diagnosis and classification. Journal of Speech, Language, and Hearing Research, 57, 57-67. 
Linblom, B. (1963). Spectrographic study of vowel reduction. Journal of the Acoustical Society of America, 35, 1773-1781.

Logemann, J., \& Fisher, H. (1981). Vocal tract control in Parkinson's disease: Phonetic feature analysis of misarticulations. Journal of Speech and Hearing Disorders, 46(4), 348-352.

Müller, J., Wenning, G., Verney, M., McKee, A., Chaudhuri, R., Jellinger, K., . . Litvan, I. (2001). Progression of dysarthria and dysphagia in postmortem-confirmed Parkinsonian disorders. Archives of neurology, 58(2), 259-264.

McRae, P., Tjaden, K., \& Schoonings, B. (2002). Acoustic and perceptual consequences of articulatory rate change in Parkinson disease. Journal of Speech, Language, and Hearing Research, 45, 35-50.

Mefferd, A. (2017). Tongue- and jaw-specific contributions to acoustic vowel contrast changes in diphthong /ai/ in response to slow, loud, and clear speech. Journal of Speech, Language, and Hearing Research, 60, 3144-3158.

Mefferd, A., \& Green, J. (2010). Articulatory-to-acoustic relations in response to speaking rate and loudness manipulations. Journal of Speech, Language, and Hearing Research, 53, 1206-1219.

Milenkovic, P. (2004). TF 32. Unpublished computer program. University of WisconsinMadison.

Nishio, M., \& Niimi, S. (2001). Speaking rate and its components in dysarthric speakers. Clinical Linguistics \& Phonetics, 4, 309-317.

Parveen, S., \& Goberman, A. (2014). Presence of stop bursts and multiple bursts in individuals with Parkinson disease. International Journal of Speech-Language Pathology, 16(5), 456-463.

Ramig, L., Fox, C., \& Sapir, S. (2004). Parkinson's disease: Speech and voice disorders and their treatment with the Lee Silverman Voice Treatment. Seminars in Speech and Language, $25,169-180$.

Ramig, L., Titze, I., Scherer, R., \& Ringel, S. (1988). Acoustic analysis of voices of patients with neurologic disease: Rationale and preliminary data. Annals of Otology, Rhinology, and Laryngology, 97, 164-172.

Sapir, S., Ramig, L., Spielman, J., \& Fox, C. (2010). Formant centralization ratio: A proposal for a new acoustic measure of dysarthric speech. Journal of Speech Language and Hearing Research, 53(1), 114-125.

Sapir, S., Spielman, J., Lorraine, R., Story, B., \& Fox, C. (2007). Effects of intensive voice treatment (the Lee Silverman Voice Treatment [LSVT]) on vowel articulation in dysarthric individuals with idiopathic Parkinson disease: Acoustic and perceptual findings. Journal of Speech Language and Hearing Research, 50, 899-912. 
Skodda, S., Visser, W., \& Schlegal, U. (2011). Vowel articulation in Parkinson's disease. Journal of Voice, 25(4), 467-472.

Skodda, S., Visser, W., \& Schlegel, U. (2009). Short- and long-term dopaminergic effects on dysarthria. Journal of Neural Transmission, 117(2), 197-205.

Smith, A., \& Kleinow, J. (2000). Kinematic correlates of speaking rate changes in stuttering and normally fluent adults. Journal of Speech, Language, and Hearing Research, 2000, 521536.

Smith, A., Johnson, M., McGillem, C., \& Goffman, L. (2000). On the assessment of stability and patterning of speech movements. Journal of Speech, Language, and Hearing Research, 43, 277-286.

Stathopoulos, E., \& Weismer, G. (1983). Closure duration of stop consonants. Journal of Phonetics, 11, 395-400.

Tjaden, K., \& Martel-Sauvageau, V. (2017). Consonant acoustics in parkinson's disease and multiple sclerosis: Comparison of clear and loud speaking conditions. American Journal of Speech-Language Pathology, 26, 569-582.

Tjaden, K., \& Wilding, G. (2004). Rate and loudness manipulations in dysarthria: Acoustic and perceptual findings. Journal of Speech, Language, and Hearing Research, 47, 766-783.

Tjaden, K., Lam, J., \& Wilding, G. (2013). Vowel acoustics in Parkinson's disease and multiple sclerosis: Comparison of clear, loud, and slow speaking conditions. Journal of Speech, Language, and Hearing Research, 56(5), 1485-1502.

Tjaden, K., Richards, E., Kuo, C., Wilding, G., \& Sussman, J. (2014). Acoustic and perceptual consequences of clear and loud speech. Folia Phoniatrica et Logopaedica, 65(4), 214220.

Tjaden, K., Sussman, J., \& Wilding, G. (2014). Impact of clear, loud, and slow speech on scaled intelligibility and speech severity in Parkinson's disease and multiple sclerosis. Journal of Speech, Language, and Hearing Research, 57, 779-792.

Walsh, B. (2007). Speech production in individuals with Parkinson's disease: Basic kinematic parameters and effects of increased linguistic demands on interarticulatory coodination. (Doctoral dissertation, Purdue University). Retrieved from http://docs.lib.purdue.edu/dissertations/ AAI3287298/.

Walsh, B., \& Smith, A. (2011). Linguistic complexity, speech production, and comprehension in Parkinson's disease: Behavioral and physiological indices. Journal of Speech, Language, and Hearing Research, 54, 787-802.

Walsh, B., \& Smith, A. (2012). Basic parameters of articulatory movements and acoustics in individuals with Parkinson's disease. Movement Disorders, 27, 843-850. 
Walsh, B., \& Smith, A. (2012). Basic parameters of articulatory movements and acoustics in individuals with Parkinson's disease. Movement Disorders, 27, 843-850.

Wang, J., Kothalker, P., Cao, B., \& Heitzman, D. (2016). Towards automatic detection of Amyotrophic Lateral Sclerosis from speech acoustic and articulatory samples. Interspeech, (pp. 1195-1199). San Francisco.

Weismer, G. (1984). Articulatory characteristics of Parkinsonian dysarthria: Segmental and phrase level timing, spirantization, and glottal-supraglottal coordination. In M. McNeal, J. Rosenback, \& A. Aronson, The dysarthrias: Physiology, acoustics, perception, management (pp. 101-130). San Diego: College-Hill Press.

Weismer, G., Jeng, J.-Y., Laures, J., Kent, R., \& Kent, J. (2001). Acoustic and intelligibillity characteristics of sentence production in neurogenic speech disorders. Folia Phoniatrica et Logopaedica, 53, 1-18.

Weismer, G., Yunusova, Y., \& Bunton, K. (2012). Measures to evaluate the effects of DBS on speech production. Journal of Neurolinguistics, 25(2), 74-94.

Westbury, J., Lindstrom, M., \& McClean, M. (2002). Tongues and lips without jaws: A comparison of methods for decoupling speech movements. Journal of Speech, Language, and Hearing Research, 45, 651-662.

Yunusova, Y., Weismer, G., Westbury, J., \& Lindstrom, M. (2008). Articulatory movements during vowels in speakers with dysarthria and healthy controls. Journal of Speech, Language, and Hearing Research, 51, 596-611. 


\section{Vita}

Austin Ross Thompson, from Mandeville, Louisiana, discovered his curiosity for speech science as an undergraduate student in the Communication Sciences and Disorders (COMD) department at Louisiana State University. As a graduate student, he solidified his passion for speech science while serving as a teaching assistant for the COMD department's Acoustics of Speech and Hearing course. Upon completion of his master's degree, he will begin to work on his doctorate under the mentorship of Dr. Yunjung Kim. 